\title{
On two extensions of the canonical Feller-Spitzer distribution
}

Vladimir Vladimirovich Vinogradov ${ }^{1 *}$ (D) and Richard Bruce Paris ${ }^{2}$

*Correspondence:

vinograd@ohio.edu

'Department of Mathematics, Ohio

University, Athens, $\mathrm{OH}$, USA

Full list of author information is

available at the end of the article

\begin{abstract}
We introduce two extensions of the canonical Feller-Spitzer distribution from the class of Bessel densities, which comprise two distinct stochastically decreasing oneparameter families of positive absolutely continuous infinitely divisible distributions with monotone densities, whose upper tails exhibit a power decay. The densities of the members of the first class are expressed in terms of the modified Bessel function of the first kind, whereas the members of the second class have the densities of their Lévy measure given by virtue of the same function. The Laplace transforms for both these families possess closed-form representations in terms of specific hypergeometric functions. We obtain the explicit expressions by virtue of the particular parameter value for the moments of the distributions considered and establish the monotonicity of the mean, variance, skewness and excess kurtosis within the families. We derive numerous properties of members of these classes by employing both new and previously known properties of the special functions involved and determine the variance function for the natural exponential family generated by a member of the second class.
\end{abstract}

Keywords: Bessel functions, Continuous-time Bernoulli random walk, Exponential convergence, Feller-Spitzer distribution, Hypergeometric functions, Kurtosis, Laplace transform, Letac-Mora reciprocity, Lévy measure, Monotone density, Natural exponential family, Power tail, Skewness, Stochastically monotone family, Variance function

Mathematics Subject Classification (2000): 60E07 60E10 60F05

\section{Introduction}

The discovery of new infinitely divisible distributions with regularly varying tails is important for the development of distribution theory per se as well as for mathematical modelling, and applications in statistics and to decision theory. Ideally, they should have tractable probability density (or mass) functions (i.e., the p.d.f. and the p.m.f., respectively) and/or Laplace transform. Here, we introduce the following two different stochastically decreasing one-parameter families of positive absolutely continuous infinitely divisible distributions whose upper tails have a power decay. 
Definition 1 Given the real-valued parameter $\rho>1 / 2$, consider the following class of positive functions of argument $x \in \mathbf{R}_{+}^{1}:=(0,+\infty)$ :

$$
f_{\rho}(x):=(2 \rho-1) \Gamma(\rho) 2^{\rho-1} \cdot \frac{e^{-x} I_{\rho}(x)}{x^{\rho}} .
$$

We define them at the origin by continuity such that

$$
f_{\rho}(0):=\lim _{x \downarrow 0} f_{\rho}(x)=(2 \rho-1) /(2 \rho) .
$$

It is shown in "Properties of the class $\mathcal{X}_{\rho}$ " section that each such function constitutes the p.d.f. of its own non-negative absolutely continuous random variable, which is hereinafter denoted by $\mathcal{X}_{\rho}$ and referred to as the generalized $\rho$-order Feller-Spitzer r.v. of the first type.

Definition 2 Consider a class of positive infinitely divisible distributions which is indexed by the real-valued parameter $\rho>1 / 2$, has the following density of its Lévy measure $v_{\rho}^{(2)}(\{\cdot\})$ on $\mathbf{R}_{+}^{1}$ :

$$
\tau_{\rho}(x):=2^{\rho-1} \cdot \Gamma(\rho) \cdot \frac{e^{-x} I_{\rho-1}(x)}{x^{\rho}} \text { with } x>0,
$$

and does not have a drift component. Note that a combination of assumption (3) with the well-known asymptotics of the modified Bessel function of the first kind $I_{r}$ (introduced by (104)) at 0 and $+\infty$ yields the fulfillment of (Bertoin (1996), condition (3.2)). Hence, such r.v., which is hereinafter denoted by $\mathcal{Y}_{\rho}$ and called the generalized $\rho$-order Feller-Spitzer r.v. of the second type, is well defined and generates its own subordinator whose corresponding Laplace transform is as follows:

$$
\begin{aligned}
\mathcal{L}_{\mathcal{Y}_{\rho}}(\lambda) & :=\mathbf{E} \exp \left\{-\lambda \mathcal{Y}_{\rho}\right\}=\exp \left\{-\Phi_{\rho}(\lambda)\right\} \\
& =\exp \left\{-\int_{0}^{\infty}\left(1-e^{-\lambda x}\right) \cdot \tau_{\rho}(x) d x\right\}, \quad \text { where } \lambda \geq 0 .
\end{aligned}
$$

For each real $\rho>1 / 2$, the non-negative r.v. $\mathcal{Y}_{\rho}$ is absolutely continuous on $\mathbf{R}_{+}^{1}$ (see Theorem 5.i). Hereinafter, we denote its p.d.f. by $p_{\rho}(x)$.

It is interesting that the Laplace transforms of members of these two new classes of distributions are related by virtue of the integral representation (60), which in turn is closely related to the concept of $\rho$-function of the generic member of a certain subclass of infinitely divisible distributions considered in (Steutel and van Harn (2004), formula (V.2.3)). (Note that the parameter $\rho$ employed throughout this paper is not related to the letter $\rho$ used in the concept of $\rho$-function due to Steutel and van Harn (2004).)

Both these families which are thoroughly studied in "Properties of the class $\mathcal{X}_{\rho}$ " and "Properties of the class $\mathcal{Y}_{\rho}$ " sections, respectively, were derived as an outgrowth of the well-known distribution presented in Definition 3 of "Review and some new results for the Feller-Spitzer class of Bessel densities" section, which is hereinafter referred to as the canonical Feller-Spitzer distribution (compare to Examples 1 and 2). This probability law first appeared in (Spitzer (1964), p. 236), but its major properties were presented by Feller (1966a) and Feller (1966b) who termed a wider class the Bessel densities.

It should be pointed out that (Johnson et al. (1994), Section 12.4.4) considered the socalled Bessel function distributions such that the p.d.f. of the generic member of such a class involves an exponentially tilted modified Bessel function of the first type (104) with an odd value of the index (see formula (12.95) therein). However, in contrast to the p.d.f's and Lévy densities (i.e., densities of the corresponding Lévy measures) which emerge in 
this work, the power term of the p.d.f.'s from (Johnson et al. (1994), Section 12.4.4) has a positive exponent, whereas it is negative in our case. Hence, the Bessel function distributions considered in (Johnson et al. (1994), Section 12.4.4) should not be confused with ours. See also "Review and some new results for the Feller-Spitzer class of Bessel densities" section for more detail on the terminology.

Each member of the two families introduced in Definitions 1-2 can be used as a mixing measure to generalize the corresponding Poisson mixture with a comparable tail behavior of its p.m.f. However, a detailed consideration of their properties is beyond the scope of this paper. See also (Paris and Vinogradov (2020a), formula (1.2)).

In this paper, we will frequently utilize several special functions including the modified Bessel function of the first kind given by (104) as well as the Gauss hypergeometric function and its generalization, which are introduced by Definitions 4 and 5, respectively. For the reader's convenience, numerous definitions and results on special functions employed in this paper are deferred to "Appendix" section. In particular, it contains two new inequalities (108) and (109) for Bessel functions.

This article is not self-contained. Hence, we refer to Letac (1992) and Jørgensen (1997) for a comprehensive description and important examples of natural exponential families (or NEF's), and to Olver et al. (2010) for more detailed information on the relevant special functions. The proof of some subtle analytic results on new properties of the modified Bessel function of the first kind (104) including the inequalities (108) and (109) are given in our work Paris and Vinogradov (2020b).

To conclude the Introduction, we summarize some notation and terminology that will be used in the sequel. First, we follow the custom of formulating various statements of distribution theory in terms of the properties of r.v.s, even when such results pertain only to their distributions. The acronym "ch.f." is used for a characteristic function. In what follows, the sign " $=$ " will denote the fact that the distributions of (univariate) r.v.s coincide, whereas the symbol “ $\stackrel{d}{\rightarrow}$ ” will stand for weak convergence. In the sequel, log stands for the natural logarithm of the real or complex argument (In the complex case, $\log z$ is understood as its principal value). An empty sum is interpreted as zero.

\section{Review and some new results for the Feller-Spitzer class of Bessel densities}

First, we present the following analytic result that can be derived from Feller (1966a).

Lemma 1 Given real $\lambda \geq 0$,

$$
\begin{aligned}
\int_{0}^{\infty} & \left(1-e^{-\lambda x}\right) \cdot \frac{e^{-x} I_{0}(x)}{x} \cdot d x \equiv-\log \int_{0}^{\infty} e^{-\lambda x} \cdot \frac{e^{-x} I_{1}(x)}{x} \cdot d x \\
& =-\log \left(\frac{1}{2(\lambda+1)} \cdot{ }_{2} F_{1}\left(\frac{1}{2}, 1 ; 2 ; \frac{1}{(\lambda+1)^{2}}\right)\right) .
\end{aligned}
$$

The above lemma will be used to relate formulas (6), (7) and (8). Separate parts of (5) give an outgrowth to Definitions 1-2 as well as the main concepts of "Properties of the class $\mathcal{X}_{\rho}$ " and "Properties of the class $\mathcal{Y}_{\rho}$ " sections.

It is known that the identity (5) is closely related to an important positive absolutely continuous infinitely divisible distribution which we call the canonical Feller-Spitzer distribution, since it was first considered by (Spitzer (1964), p. 236), Feller (1966a), and Feller (1966b). It is introduced as follows: 
Definition 3 The canonical Feller-Spitzer positive absolutely continuous r.v. $\mathcal{U}_{1}$ is defined by virtue of its $p . d . f$.

$$
f_{1}(x):=x^{-1} e^{-x} I_{1}(x), \text { where } x \in \mathbf{R}_{+}^{1} .
$$

By Feller (1966a) and Feller (1966b), r.v. $\mathcal{U}_{1}$ is infinitely divisible having the following density $\tau_{1}(x)$ of its Lévy measure (or its Lévy density):

$$
\tau_{1}(x) \equiv x^{-1} e^{-x} I_{0}(x), \text { where } x \in \mathbf{R}_{+}^{1} .
$$

In view of (5), the Laplace transform of $\mathcal{U}_{1}$ acquires the following form for $\lambda \geq 0$ :

$$
\begin{aligned}
& \mathcal{L}_{\mathcal{U}_{1}}(\lambda):=\mathbf{E} e^{-\lambda \mathcal{U}_{1}}=\exp \left\{-\int_{0}^{\infty}\left(1-e^{-\lambda x}\right) \tau_{1}(x) d x\right\} \\
& =\lambda+1-\sqrt{\lambda^{2}+2 \lambda}=\frac{1}{2(\lambda+1)} \cdot{ }_{2} F_{1}\left(\frac{1}{2}, 1 ; 2 ; \frac{1}{(\lambda+1)^{2}}\right) .
\end{aligned}
$$

The variance function $\mathbf{V}_{1}(\mu)$ of the NEF constructed starting from the canonical FellerSpitzer r.v. $\mathcal{U}_{1}$ with p.d.f. $f_{1}(x)$ is as follows:

$$
\mathbf{V}_{1}(\mu)=\mu^{2} \cdot \sqrt{\mu^{2}+1} \text {, where } \mu \in(0,+\infty] .
$$

The validity of (9) was stated by (Letac (1987), p. 154) in his discussion of (Jørgensen (1987), Example 2.1). This also follows from a combination of (Jørgensen (1997), formula (2.18)) with the fact that the inverse of the mean-value mapping for a NEF generated by r.v. $\mathcal{U}_{1}$ equals

$$
1-\sqrt{1+1 / \mu^{2}} \leq 0 \text { with } \mu \in(0,+\infty] .
$$

We refer to (Jørgensen (1997), p. 48) for more detail on the variance function of a generic $\mathrm{NEF}$ and a related concept of the mean-value mapping.

The motivation behind the consideration of r.v. $\mathcal{U}_{1}$ in (Spitzer (1964), p. 236) and (Feller (1966a), Subsection II.7.b) was partly because it emerges as the law of the first passage time of level 1 for the so-called continuous-time symmetric Bernoulli random walk which is characterized by mean 1 exponential time in between the jumps. More specifically, if $\left\{\mathcal{B}_{\ell}, \ell \geq 1\right\}$ are i.i.d.r.v.s such that $\mathbf{P}\left\{\mathcal{B}_{\ell}= \pm 1\right\}=1 / 2$ independent of a Poisson process $\{\mathcal{N}(t), t \geq 0\}$ with unit intensity, consider the (continuous-time and discrete space) compound Poisson Lévy process $\mathcal{S}(t) \stackrel{\mathrm{d}}{=} \mathcal{B}_{1}+\ldots+\mathcal{B}_{\mathcal{N}(t)}$ and the corresponding first passage time of positive integer level $n \geq 1$ :

$$
\mathcal{T}_{n}:=\min (t \geq 0: \mathcal{S}(t)=n) .
$$

Then the r.v. $\mathcal{T}_{n}$ has the p.d.f. $f_{1}^{* n}(u)$ of the $n^{\text {th }}$ partial $\operatorname{sum} \mathcal{U}_{1}(1)+\ldots+\mathcal{U}_{1}(n)$ of $n$ i.i.d.r.v.s with common canonical Feller-Spitzer distribution $\mathcal{U}_{1}$ with the p.d.f. $f_{1}(u)$.

The p.d.f. $f_{1}^{* t}(u)$ was found by (Spitzer (1964), p. 236), Feller (1966a); Feller (1966b) to be as follows:

$$
f_{1}^{* t}(u)=\frac{t}{u} \cdot e^{-u} I_{t}(u), \quad \text { where } u \in \mathbf{R}_{+}^{1} .
$$

(It is straightforward to check that the expression which emerges on the right-hand side of (11) constitutes a legitimate p.d.f. on $\mathbf{R}_{+}^{1}$ for an arbitrary real-valued $t \in \mathbf{R}_{+}^{1}$.)

The cumulant-generating function (or the c.g.f.) of the corresponding compound Poisson-Bernoulli r.v. $\mathcal{S}(1)$ equals

$$
\cosh \theta-1=\left(e^{\theta}+e^{-\theta}\right) / 2-1 \text { where } \theta \in \mathbf{R}^{1} .
$$


Subsequently, in view of a slight modification of (Jørgensen (1997), pp. 124-125, Exercise 3.15.4), the variance function of the NEF constructed starting from the r.v. with c.g.f. (12) equals

$$
\sqrt{\mu^{2}+1} \text {, where } \mu \in \mathbf{R}^{1} \text {. }
$$

Remark 1 It is straightforward to verify that the pair of the variance functions given by formulas (13) and (9) constitutes the reciprocal pair in the sense of (Letac and Mora (1990), Theorem 5.2.iii). In turn, this is consistent with a comment concerning the fluctuation properties of the right-continuous random walk in Spitzer's sense, which was made in the paragraph that precedes (Letac and Mora (1990), Theorem 5.6). See also (Spitzer (1964), Definition 1.2.3) for the definition of the latter random walk as well as a comment that follows this definition.

Note in passing that Feller considered a more general class of p.d.f.'s on $\mathbf{R}_{+}^{1}$, some of which became important in applications. For instance, it contains the distribution of the busy period in $M / M / 1$ queue (compare to (Stewart (2009), p. 530)).

\section{Properties of the class $\mathcal{X}_{\rho}$}

First, recall that this class was introduced in Definition 1 of "Introduction" section.

Next, the integrability of $f_{\rho}(x)$ to one follows from the fact that in the case where $s=0$, the expression that emerges on the right-hand side of (39) equals 1 . Hence, $f_{\rho}(x)$ is a legitimate p.d.f. By (Olver et al. (2010), formula (10.30.4))), it exhibits the following power decay as $x \rightarrow+\infty$

$$
f_{\rho}(x) \sim \frac{(2 \rho-1) 2^{\rho-3 / 2}}{\sqrt{\pi}} \cdot \frac{\Gamma(\rho)}{x^{\rho+1 / 2}} .
$$

It follows from the closed-form representations (1)-(2) with the help of Mathematica that for arbitrary fixed $\rho>1 / 2$ and $y \in[0,+\infty)$,

$$
\mathbf{P}\left\{\mathcal{X}_{\rho} \leq y\right\}=\int_{0}^{y} f_{\rho}(x) \cdot d x=1-{ }_{1} F_{1}(\rho-1 / 2 ; 2 \rho ;-2 y) .
$$

Hereinafter ${ }_{1} F_{1}(\cdot ; \cdot ; \cdot)$ denotes the confluent hypergeometric function (see, for example, (Slater (1960), formula (1.1.8))).

The following assertion stipulates that the family $\left\{\mathcal{X}_{\rho}, \rho>1 / 2\right\}$ possesses an important property of stochastic monotonicity. The interested reader is referred to Cohn (1981) and Lee et al. (2009) for a connection between this concept and various convergence properties, and for relevant statistical tests and applications, respectively (see also the references therein). Its relationship to weak convergence is illustrated just above Propositions 3 and 7. Also, note in passing that in the context of a family of positive r.v.s, stochastic monotonicity is equivalent to monotonicity of the corresponding family of the survival functions at each value of the argument.

Theorem 1 The family $\left\{\mathcal{X}_{\rho}, \rho>1 / 2\right\}$ of the generalized Feller-Spitzer distributions of the first type is stochastically decreasing in the sense that for an arbitrary fixed real $y>0$, the function $\rho \rightarrow \mathbf{P}\left\{\mathcal{X}_{\rho}>y\right\}$ is decreasing on $(1 / 2, \infty)$. 
Proof of Theorem 1. In view of the above comment, it suffices to prove monotonicity of the family of the values of the survival functions for each value of argument $y \in \mathbf{R}_{+}^{1}$. A subsequent combination of formula (15) with Kummer's transformation (see (Olver et al. (2010), formula (13.2.39)) yields that for arbitrary fixed $\rho>1 / 2$ and $y \in[0,+\infty)$, the survival function $\mathcal{F}_{\rho}(y)$ of the r.v. $\mathcal{X}_{\rho}$ admits the following representation:

$$
\begin{gathered}
\mathcal{F}_{\rho}(y):=\mathbf{P}\left\{\mathcal{X}_{\rho}>y\right\}={ }_{1} F_{1}\left(\rho-\frac{1}{2} ; 2 \rho ;-2 y\right) \\
=e^{-2 y}{ }_{1} F_{1}\left(\rho+\frac{1}{2} ; 2 \rho ; 2 y\right)=e^{-2 y} \sum_{n=0}^{\infty} t_{n}(\rho) \frac{(2 y)^{n}}{n !},
\end{gathered}
$$

where $t_{n}(\rho):=(\rho+1 / 2)_{n} /(2 \rho)_{n}>0$. Then we have

$$
\log t_{n}(\rho)=\sum_{k=0}^{n-1} \log \left(\frac{\rho+k+1 / 2}{2 \rho+k}\right)
$$

so that

$$
\frac{d}{d \rho} \log t_{n}(\rho)=\frac{t_{n}^{\prime}(\rho)}{t_{n}(\rho)}=\sum_{k=0}^{n-1} \frac{2 \rho+k}{\rho+k+1 / 2} \cdot \frac{d}{d \rho}\left(\frac{\rho+k+1 / 2}{2 \rho+k}\right)<0
$$

since

$$
\frac{d}{d \rho}\left(\frac{\rho+k+\frac{1}{2}}{2 \rho+k}\right)=-\frac{k+1}{(2 \rho+k)^{2}}<0 .
$$

Finally, as the sum in $\mathcal{F}_{\rho}(y)$ consists of positive terms, and the coefficients $t_{n}(\rho)$ are positive decreasing functions of $\rho$, it follows that the survival function $\mathcal{F}_{\rho}(y)$ must decrease with increasing $\rho$.

Proposition 1 For each $\rho>1 / 2$, the Laplace transform of r.v. $\mathcal{X}_{\rho}$ is such that for $\lambda \in$ $[0,+\infty)$

$$
\mathcal{L}_{\mathcal{X}_{\rho}}(\lambda):=\mathbf{E} \exp \left\{-\lambda \cdot \mathcal{X}_{\rho}\right\}=\frac{2 \rho-1}{2 \rho} \cdot \frac{1}{\lambda+1} \cdot{ }_{2} F_{1}\left(\frac{1}{2}, 1 ; \rho+1 ; \frac{1}{(\lambda+1)^{2}}\right) .
$$

Proof of Proposition 1. First, the following integral representation for the function ${ }_{2} F_{1}$ can be derived with some effort from (Watson (1952), Section 13.2, p. 385, formula (2)) by implementing the change of variables $\mathbf{b} \rightarrow i \mathbf{b}$ in this formula and using the fact that the Bessel function of the first kind $J_{r}(\mathbf{i} \mathbf{b} t)=e^{\pi r i / 2} I_{r}(\mathbf{b} t)$ :

$$
{ }_{2} F_{1}\left(\frac{1}{2} k, \frac{1}{2} k+\frac{1}{2} ; \mathbf{c} ; x\right)=\frac{\mathbf{a}^{k}(\mathbf{b} / 2)^{1-\mathbf{c}} \Gamma(\mathbf{c})}{\Gamma(k)} \cdot \int_{0}^{\infty} e^{-\mathbf{a} t} I_{\mathbf{c}-1}(\mathbf{b} t) \cdot t^{k-\mathbf{c}} d t .
$$

Here, $\mathbf{a}>\mathbf{b}>0, \mathbf{c} \neq 0,-1,-2, \ldots$, integer $k \geq 1$, and $x=\mathbf{b}^{2} / \mathbf{a}^{2} \in(0,1)$.

A subsequent combination of (18) with the values of $\mathbf{a}=1, \mathbf{b}=1 /(\lambda+1), \mathbf{c}=\rho+1$, $k=1$ and (1)-(2) implies the validity of (17). Compare to the derivation of (Paris and Vinogradov (2020a), formula (5.13)) where the case of $\mathbf{c}=2$ was considered.

Evidently, the closed-form expression which emerges in (17) generalizes formula (8) which pertains to the case where $\rho=1$.

Example 1 For $\rho=1$, formula (1) turns into (6). Hence, the distributions of r.v. $\mathcal{X}_{1}$ and the canonical Feller-Spitzer r.v. $\mathcal{U}_{1}$ coincide, i.e.,

$$
\mathcal{X}_{1} \stackrel{\mathrm{d}}{=} \mathcal{U}_{1} \text {. }
$$


It is clear that in the case where $\rho=2$, the p.d.f. of the r.v. $\mathcal{X}_{2}$ is such that for $x \in \mathbf{R}_{+}^{1}$,

$$
f_{2}(x)=6 e^{-x} I_{2}(x) / x^{2} \rightarrow 3 / 4 \text { as } x \downarrow 0 .
$$

Also, a combination of (17) with (Paris and Vinogradov (2020a), formula (4.1)) yields that for a fixed $\lambda \in[0,+\infty)$,

$$
\mathcal{L}_{\mathcal{X}_{2}}(\lambda)=3(\lambda+1)-2(\lambda+1)^{3}+2\left(\lambda^{2}+2 \lambda\right)^{3 / 2} .
$$

It is relevant that by analogy with (21), it is possible to derive the closed-form representations for $\mathcal{L}_{X_{\rho}}(\lambda)$ for numerous other values of $\rho>1 / 2$ some of which follow from (Paris and Vinogradov (2020a), formula (4.1)).

Theorem 2 Fix an arbitrary real $\rho>1 / 2$. Then (i) The r.v. $\mathcal{X}_{\rho}$ is infinitely divisible.

(ii) For $x \in \mathbf{R}_{+}^{1}$, one ascertains that the p.d.f. $f_{\rho}(\cdot) \in \mathbf{C}^{\infty}\left(\mathbf{R}_{+}^{1}\right)$ such that

$$
f_{\rho}^{\prime}(x)=(2 \rho-1) \Gamma(\rho) 2^{\rho-1} \cdot e^{-x} \cdot \frac{I_{\rho+1}(x)-I_{\rho}(x)}{x^{\rho}}<0,
$$

and

$$
f_{\rho}^{\prime \prime}(x)=(2 \rho-1) \Gamma(\rho) 2^{\rho} \frac{e^{-x}}{x^{\rho}}\left\{I_{\rho}(x)-\left(1+\frac{2 \rho+1}{2 x}\right) I_{\rho+1}(x)\right\}>0 .
$$

(iii) The p.d.f. $f_{\rho}(x)$ decreases from $(2 \rho-1) /(2 \rho)$ to 0 on $[0,+\infty)$.

(iv) The values of the p.d.f. $f_{\rho}(x)$ and its first derivative at the origin are only different by the sign such that

$$
f_{\rho}^{\prime}(0+)=-f_{\rho}(0)=(1-2 \rho) /(2 \rho) .
$$

(v) The following recursive formula holds for $x \in \mathbf{R}_{+}^{1}$ :

$$
f_{\rho+1}(x)=\frac{2 \rho \cdot(2 \rho+1)}{2 \rho-1} \cdot \frac{f_{\rho}^{\prime}(x)+f_{\rho}(x)}{x} .
$$

Proof of Theorem 2. (i) It follows from the fact that for $\rho>1 / 2$, the distribution of the r.v. $\mathcal{X}_{\rho}$ satisfies the log-convexity property, which easily follows from inequality (108) of Theorem 8.i (see Corollary 1 of "Appendix" section for more detail).

It remains to apply (Steutel and van Harn (2004), pp. 117-118, Th. III.10.2) which stipulates that log-convexity is sufficient for a distribution on $\mathbf{R}_{+}^{1}$ to be infinitely divisible.

(ii) The validity of (22) easily follows from a combination of (110) and (112).

Also, note that it immediately follows from formula (109) (with $v$ replaced by $\rho$ ) that $f_{\rho}^{\prime \prime}(x)>0$ for $x>0$ and $\rho>1 / 2$ thus establishing (23).

(iii) The proof of (24) follows from a combination of (2) and (22).

(iv) Let us replace $\rho$ by $\rho+1$ in (1) and combine the corresponding representation for $f_{\rho+1}(x)$ with (Paris and Vinogradov (2020b), formula (4.4) and the unnumbered formula just below (4.6)). We obtain that

$$
\begin{aligned}
& f_{\rho+1}(x)=(2 \rho+1) \cdot \Gamma(1+\rho) \cdot 2^{\rho} \cdot \frac{e^{-x}}{x} \cdot \frac{I_{\rho+1}(x)}{x^{\rho}} \\
& =(2 \rho+1) \cdot \Gamma(1+\rho) \cdot 2^{\rho} \cdot\left(e^{-x} / x\right) \cdot\left(I_{\rho}(x) / x^{\rho}\right)^{\prime} .
\end{aligned}
$$

In view of (Paris and Vinogradov (2020b), formula (4.5)), the expression which emerges on the right-hand side of the above equation equals

$$
(2 \rho+1) \cdot \Gamma(1+\rho) \cdot 2^{\rho} \cdot x^{-1} \cdot\left\{\left(e^{-x} I_{\rho}(x) / x^{\rho}\right)^{\prime}+e^{-x} I_{\rho}(x) / x^{\rho}\right\} .
$$

The result then follows upon insertion of the definition (1) of $f_{\rho}(x)$. 
Proposition 2 (i) The canonical Feller-Spitzer r.v. $\mathcal{U}_{1} \stackrel{\mathrm{d}}{=} \mathcal{X}_{1}$ can be decomposed into the sum of two independent non-negative infinitely divisible rv's one of which is the generalized 2-order Feller-Spitzer r.v. of the first type $\mathcal{X}_{2}$, and the other r.v. $\mathcal{V}$ which is characterized by its Laplace transform

$$
\begin{aligned}
& \mathcal{L}_{\mathcal{V}}(\lambda):=\mathbf{E} \exp \{-\lambda \cdot \mathcal{V}\}=\mathcal{L}_{\mathcal{X}_{1}}(\lambda) / \mathcal{L}_{\mathcal{X}_{2}}(\lambda) \\
& =\frac{\lambda+1-\sqrt{\lambda^{2}+2 \lambda}}{3(\lambda+1)-2(\lambda+1)^{3}+2\left(\lambda^{2}+2 \lambda\right)^{3 / 2}}
\end{aligned}
$$

with $\lambda \in[0,+\infty)$, such that

$$
\mathcal{U}_{1} \stackrel{\mathrm{d}}{=} \mathcal{X}_{1} \stackrel{\mathrm{d}}{=} \mathcal{X}_{2}+\mathcal{V} \text {. }
$$

(ii) The r.v. $\mathcal{V}$ admits the following compound geometric representation:

$$
\mathcal{V} \stackrel{\mathrm{d}}{=} \mathcal{W}_{1}+\ldots+\mathcal{W}_{\mathcal{G} e o m(2 / 3)},
$$

where $\left\{\mathcal{W}_{\ell}, \ell \geq 1\right\}$ are positive i.i.d.r.v's having common Laplace transform

$$
\mathcal{L}_{\mathcal{W}_{\ell}}(\lambda)=\mathcal{L}_{\mathcal{U}_{1}}(\lambda)^{2}=\left(\lambda+1-\sqrt{\lambda^{2}+2 \lambda}\right)^{2} \text { for } \lambda \geq 0
$$

and the p.d.f.

$$
f_{\mathcal{W}_{\ell}}(u) \equiv f_{1}^{* 2}(u)=\frac{2}{u} \cdot e^{-u} \cdot I_{2}(u) \quad\left(\text { with } u \in \mathbf{R}_{+}^{1}\right)
$$

independent of a geometric counting r.v. Geom(2/3) (that takes values in the set of all nonnegative integers), which is characterized by its Laplace transform

$$
\begin{aligned}
& \mathcal{L}_{\mathcal{G} \text { eom }(2 / 3)}(\lambda):=\mathbf{E} \exp \{-\lambda \cdot \mathcal{G} \operatorname{eom}(2 / 3)\} \\
& =\frac{2 / 3}{1-1 /\left(3 e^{\lambda}\right)}, \quad \text { where } \lambda>-\log 3 .
\end{aligned}
$$

Hereinafter, the p.d.f. $f_{1}^{* 2}(u)$ which emerges in the middle of formula (30) denotes the two-fold convolution of the p.d.f. $f_{1}(u)$ of the r.v. $\mathcal{U}_{1}$ (compare to (11)).

Proof of Proposition 2. First, one should verify the following identity:

$$
\mathcal{L}_{\mathcal{V}}(\lambda) \equiv \mathcal{L}_{\mathcal{G e o m}(2 / 3)}\left(\mathcal{L}_{\mathcal{W}}(\lambda)\right) \equiv \frac{2}{3-\left(\lambda+1-\sqrt{\lambda^{2}+2 \lambda}\right)^{2}},
$$

where the Laplace transforms which emerge in identity (32) are given by formulas (26), (31) and (29). This verification is straightforward and hence left to the reader. In turn, (32) implies the validity of representation (28). The rest follows from a rather obvious folk theorem that a compound geometric sum of i.i.d.r.v.s is infinitely divisible (compare to (Kyprianou (2010), claim above formula (8)).

Although $\mathcal{X}_{\rho}$ is infinitely divisible $\forall \rho>1 / 2$, it is quite challenging to find its Lévy representation for $\rho \neq 1$. Recall that for $\rho=1$, it is given by (8).

Theorem 2.i stipulates that for a fixed real $\rho>1 / 2$, there exists the Lévy measure $v_{\rho}^{(1)}(\{\cdot\})$ on $\mathbf{R}_{+}^{1}$ of positive infinitely divisible r.v. $\mathcal{X}_{\rho}$ such that for real $\lambda \geq 0$,

$$
\log \mathcal{L}_{\mathcal{X}_{\rho}}(\lambda)=-\int_{0}^{\infty}\left(1-e^{-\lambda x}\right) \cdot v_{\rho}^{(1)}(d x) .
$$


Next, a combination of formula (15) with (Embrechts et al. (1979), Theorem 1) and (Abramowitz and Stegun (1965), formula (13.5.1)) implies that as $y \rightarrow+\infty$,

$$
\begin{aligned}
& v_{\rho}^{(1)}(\{(y, \infty)\}) \sim \mathbf{P}\left\{\mathcal{X}_{\rho}>y\right\} \\
= & { }_{1} F_{1}(\rho-1 / 2 ; 2 \rho ;-2 y) \sim \frac{2^{\rho-1 / 2}}{\sqrt{\pi}} \Gamma(\rho) y^{-(\rho-1 / 2)},
\end{aligned}
$$

where the rightmost asymptotics in (33) is consistent with (14).

In addition, for real $y>0$ the Lévy measure $v_{1}^{(1)}(\{(y, \infty)\})$ of the open ray $(y, \infty)$ of the canonical Feller-Spitzer r.v. $\mathcal{U}_{1}\left(\stackrel{\mathrm{d}}{=} \mathcal{X}_{1}\right)$ can be derived from (7) with the use of Mathematica. Specifically,

$$
\begin{gathered}
\nu_{1}^{(1)}(\{(y, \infty)\}):=\int_{y}^{\infty} x^{-1} e^{-x} I_{0}(x) d x=\log (y / 2)-\gamma \\
+y \cdot{ }_{3} F_{3}(1,1,3 / 2 ; 2,2,2 ;-2 y) \sim \sqrt{2 / \pi} \cdot y^{-1 / 2} \text { as } y \rightarrow+\infty .
\end{gathered}
$$

Hereinafter, $\gamma$ denotes the Euler-Mascheroni constant (see (106)).

Note in passing that in view of (34) and as $y \rightarrow+\infty$,

$$
v_{1}^{(1)}(\{(y, \infty)\}) \sim \sqrt{2 / \pi} \cdot y^{-1 / 2} \sim \mathbf{P}\left\{\mathcal{U}_{1}>y\right)=\int_{y}^{\infty} f_{1}(x) d x,
$$

which is consistent with (33) and (Embrechts et al. (1979), Theorem 1).

We believe that representation (34) for the Lévy measure $v_{1}^{(1)}(\{(y, \infty)\})$ in terms of the function ${ }_{3} F_{3}$ was previously unknown. See also Proposition 6, which extends formulas (34)-(35) for all the $\rho$-order Feller-Spitzer r.v.s of the second type introduced in Definition 2. Also, both a closed-form representation for the cumulative distribution function of r.v. $\mathcal{U}_{1}$ in terms of the confluent hypergeometric function ${ }_{1} F_{1}$ and the asymptotics of its upper tail can be derived by setting $\rho=1$ in formulas (15) and (33), respectively.

Next, Theorem 1 implies with some effort existence of the weak limit for the stochastically decreasing family $\mathcal{X}_{\rho}$ as $\rho \rightarrow+\infty$, which should be a continuous distribution on $\mathbf{R}_{+}^{1}$. In turn, part (i) of the following assertion identifies this limit as the mean 1 exponential r.v., which is hereinafter denoted by $\mathcal{E}$, whereas part (ii) can be regarded as a local limit theorem on the exponential convergence.

Proposition 3 Suppose that $\rho \rightarrow+\infty$. Then

(i)

$$
\mathcal{X}_{\rho} \stackrel{\mathrm{d}}{\rightarrow} \mathcal{E} .
$$

(ii) For a fixed $z \in[0,+\infty)$,

$$
f_{\rho}(z) \rightarrow e^{-z}
$$

Proof of Proposition 3. (i) It follows from (17) that for an arbitrary fixed $\lambda \in[0,+\infty)$ and as $\rho \rightarrow+\infty$,

$$
\mathcal{L}_{\mathcal{X}_{\rho}}(\lambda) \rightarrow 1 /(\lambda+1) .
$$

The result follows, since the limit $1 /(\lambda+1)$ which emerges on the right-hand side of (38) coincides with the Laplace transform of $\mathcal{E}$.

(ii) It is easily derived by combining (1)-(2) with (Olver et al. (2010), formula (10.19.1)) and Stirling's formula for the gamma function. 
Next, it easily follows from a combination of (6) with formula (107) of Lemma 3 that given real $s \in(-1, \rho-1 / 2)$, the $s^{\text {th }}$ raw moment of the r.v. $\mathcal{X}_{\rho}$ is as follows:

$$
\mathbf{E}\left(\mathcal{X}_{\rho}^{s}\right):=\int_{0}^{\infty} x^{s} \cdot f_{\rho}(x) d x=\frac{2 \rho-1}{2} \cdot \frac{\Gamma(s+1) \Gamma(\rho) \Gamma(\rho-s-1 / 2)}{\Gamma(\rho-s / 2) \Gamma(\rho-s / 2+1 / 2)} .
$$

Subsequently, we employ the above formula (39) to provide the closed-form representations for the mean, variance, skewness and excess kurtosis for those members of the class $\left\{X_{\rho}, \rho>1 / 2\right\}$ for which the corresponding numerical characteristic(s) are finite.

Proposition 4 (i) Given $\rho>3 / 2$, the mean

$$
\mu_{\rho}^{(1)}:=\mathbf{E} \mathcal{X}_{\rho}=\frac{2 \rho-1}{2 \rho-3}
$$

decreases from $+\infty$ to 1 as $\rho$ increases from $3 / 2$ to $+\infty$.

(ii) The second, third and fourth central moments of r.v. $\mathcal{X}_{\rho}$ admit the following closedform representations.

For $\rho>5 / 2$, the variance

$$
\left(\sigma_{\rho}^{(1)}\right)^{2}:=\operatorname{Var} \mathcal{X}_{\rho}=\frac{(2 \rho-1)\left(4 \rho^{2}-8 \rho+7\right)}{(2 \rho-3)^{2}(2 \rho-5)}
$$

decreases from $+\infty$ to 1 as $\rho$ increases from $5 / 2$ to $+\infty$.

Given $\rho>7 / 2$,

$$
\begin{aligned}
& \mu_{3}\left(\mathcal{X}_{\rho}\right):=\mathbf{E}\left(\left(\mathcal{X}_{\rho}-\mu_{\rho}^{(1)}\right)^{3}\right) \\
& =\frac{2(2 \rho-1)\left(16 \rho^{4}-64 \rho^{3}+144 \rho^{2}-152 \rho+71\right)}{(2 \rho-3)^{3}(2 \rho-5)(2 \rho-7)} .
\end{aligned}
$$

Given $\rho>9 / 2$,

$$
\begin{gathered}
\mu_{4}\left(\mathcal{X}_{\rho}\right):=\mathbf{E}\left(\left(\mathcal{X}_{\rho}-\mu_{\rho}^{(1)}\right)^{4}\right) \\
=\frac{3(2 \rho-1)\left(192 \rho^{6}-1408 \rho^{5}+5104 \rho^{4}-10368 \rho^{3}+12548 \rho^{2}-8456 \rho+2493\right)}{(2 \rho-3)^{4}(2 \rho-5)(2 \rho-7)(2 \rho-9)} .
\end{gathered}
$$

Proof of Proposition 4. It follows with sufficient effort from (39) with the help of Mathematica.

An application of the representations of Proposition 4.ii with the help of Mathematica stipulates that the skewness $\Gamma_{1}^{(1)}(\rho)$ and the excess kurtosis $\Gamma_{2}^{(1)}(\rho)$ of the r.v. $\mathcal{X}_{\rho}$ are as follows. For $\rho>7 / 2$, the skewness

$$
\begin{aligned}
& \Gamma_{1}^{(1)}(\rho):=\mu_{3}\left(\mathcal{X}_{\rho}\right) /\left(\sigma_{\rho}^{(1)}\right)^{3} \\
& =\frac{2(2 \rho-5)^{1 / 2}\left(16 \rho^{4}-64 \rho^{3}+144 \rho^{2}-152 \rho+71\right)}{(2 \rho-1)^{1 / 2}(2 \rho-7)\left(4 \rho^{2}-8 \rho+7\right)^{3 / 2}}
\end{aligned}
$$

decreases from $+\infty$ to 2 as $\rho$ increases from $7 / 2$ to $+\infty$. 
Also, for $\rho>9 / 2$, the excess kurtosis

$$
\begin{gathered}
\Gamma_{2}^{(1)}(\rho):=\mu_{4}\left(\mathcal{X}_{\rho}\right) /\left(\sigma_{\rho}^{(1)}\right)^{4}-3 \\
=3\left\{\frac{(2 \rho-5)\left(192 \rho^{6}-1408 \rho^{5}+5104 \rho^{4}-10368 \rho^{3}+12548 \rho^{2}-8456 \rho+2493\right)}{(2 \rho-1)(2 \rho-7)(2 \rho-9)\left(4 \rho^{2}-8 \rho+7\right)^{2}}\right. \\
-1\}
\end{gathered}
$$

decreases from $+\infty$ to 6 as $\rho$ increases from $9 / 2$ to $+\infty$.

Remark 2 (i) The representations (40), (41), (44) and (45) stipulate that as $\rho \rightarrow+\infty$, the mean, variance, skewness and excess kurtosis of the respective members of the class $\left\{\mathcal{X}_{\rho}\right\}$ converge to 1, 1, 2 and 6, respectively, which are the values of the corresponding numerical characteristics of the mean 1 exponential r.v. $\mathcal{E}$. This observation on the moment convergence is consistent with the integral and local theorems on the exponential convergence presented in Proposition 3 above.

(ii) In view of (44), the subclass $\left\{\mathcal{X}_{\rho}, \rho>7 / 2\right\}$ is comprised of the right skewed distributions. In turn, (45) stipulates that each member of the subclass $\left\{\mathcal{X}_{\rho}, \rho>9 / 2\right\}$ is leptokurtic.

\section{Properties of the class $\mathcal{Y}_{\rho}$}

First, recall that this class was introduced in Definition 2 of "Introduction" section. This second extension of the canonical Feller-Spitzer distribution $\mathcal{U}_{1}$ (introduced in Definition 3) concerns its Lévy density $\tau_{1}(x)$ given by (7), which is in contrast to the first extension $\left\{f_{\rho}(x), \rho>1 / 2\right\}$ which pertains to the p.d.f. $f_{1}(x)$ per se.

Next, a combination of (3) with (Olver et al. (2010), formula (10.30.4))) implies that the Lévy density $\tau_{\rho}(x)$ (which is introduced by formula (3)) exhibits the following power decay as $x \rightarrow+\infty$ :

$$
\tau_{\rho}(x) \sim \frac{2^{\rho-3 / 2}}{\sqrt{\pi}} \cdot \frac{\Gamma(\rho)}{x^{\rho+1 / 2}}
$$

(compare to (14) and (71)). On the other hand, it follows with some effort from a combination of (3) with (Olver et al. (2010), formulas (10.25.2) and (10.30.1))) that this function diverges at the origin exhibiting the following asymptotic behavior at the right neighborhood of zero:

$$
\tau_{\rho}(x)=\frac{1}{x}-1+\frac{1+2 \rho}{4 \rho} \cdot x-\frac{3+2 \rho}{12 \rho} \cdot x^{2}+\mathcal{O}\left(x^{3}\right) \quad \text { as } x \downarrow 0 .
$$

The following result is similar in spirit to Theorem 1 of "Properties of the class $\mathcal{X}_{\rho}$ " section.

Theorem 3 The family $\left\{\mathcal{Y}_{\rho}, \rho>1 / 2\right\}$ of the generalized Feller-Spitzer distributions of the second type is stochastically decreasing in the sense that for an arbitrary fixed real $y>0$, the function $\rho \rightarrow \mathbf{P}\left\{\mathcal{Y}_{\rho}>y\right\}$ is decreasing on $(1 / 2, \infty)$.

In order to prove Theorem 3, we first present the following general and straightforward but rather technical assertion which might already be known. 
Lemma 2 Suppose that for $i=1,2$ there exist two positive functions $\tau^{(i)}(\cdot)$ on $\mathbf{R}_{+}^{1}$ such that the following integrals are well-defined and finite:

$$
\int_{0}^{\infty} \min (1, x) \cdot \tau^{(i)}(x) \cdot d x<\infty .
$$

Let $\tau^{(1)}(x)>\tau^{(2)}(x)$ on $\mathbf{R}_{+}^{1}$. Assume that for $i=1,2$ there exist two non-negative infinitely divisible r.v's $\mathbf{Y}_{i}$ with zero drifts and diffusion components which possess the Lévy densities on $\mathbf{R}_{+}^{1}$ given by functions $\tau^{(i)}(\cdot)$, respectively, such that for real $\lambda \geq 0$,

$$
\mathcal{L}_{\mathbf{Y}_{i}}(\lambda):=\mathbf{E} e^{-\lambda \cdot \mathbf{Y}_{i}}=\exp \left\{-\int_{0}^{\infty}\left(1-e^{-\lambda x}\right) \cdot \tau^{(i)}(x) \cdot d x\right\} .
$$

Then

(i) There exists a non-negative infinitely divisible r.v. $\mathcal{K}$ independent of $\mathbf{Y}_{2}$ such that

$$
\mathbf{Y}_{1} \stackrel{\mathrm{d}}{=} \mathbf{Y}_{2}+\mathcal{K} \text {. }
$$

(ii) For an arbitrary fixed $y \in \mathbf{R}_{+}^{1}$,

$$
\mathbf{P}\left\{\mathbf{Y}_{1} \leq y\right\} \leq \mathbf{P}\left\{\mathbf{Y}_{2} \leq y\right\} .
$$

\section{Proof of Lemma 2.}

(i) It follows from the assumptions of the lemma and (49) that for real $\lambda \geq 0$,

$$
-\left(\log \mathcal{L}_{\mathbf{Y}_{1}}(\lambda)-\log \mathcal{L}_{\mathbf{Y}_{2}}(\lambda)\right)=\int_{0}^{\infty}\left(1-e^{-\lambda x}\right) \cdot\left(\tau^{(1)}(x)-\tau^{(2)}(x)\right) d x>0 .
$$

In view of (48)-(49), the expression which emerges on the left-hand side of (52) constitutes the negative of the log-Laplace transform of a certain non-negative infinitely divisible r.v. denoted by $\mathcal{K}$ whose density of Lévy measure equals $\tau^{(1)}(x)-\tau^{(2)}(x)$, which implies (50).

(ii) The validity of (51) easily follows from (50).

Proof of Theorem 3. First, let us prove that for a fixed $x \in \mathbf{R}_{+}^{1}$, the Lévy density $\tau_{\rho}(x)$ defined by (3) is a decreasing function of the parameter $\rho$ on $(1 / 2,+\infty)$. To this end, we rewrite this function as follows:

$$
\begin{aligned}
& \tau_{\rho}(x)=2^{\rho-1} \Gamma(\rho) \frac{e^{-x} I_{\rho-1}(x)}{x^{\rho}} \\
= & 2^{\rho-1} \Gamma(\rho) \frac{e^{-x}}{x^{\rho}}\left(\frac{1}{2} x\right) \sum_{k \geq 0}^{\rho-1} \frac{\left(\frac{1}{2} x\right)^{2 k}}{k ! \Gamma(k+\rho)}=\frac{e^{-x}}{x} \sum_{k \geq 0} \frac{\left(\frac{1}{2} x\right)^{2 k}}{k !(\rho)_{k}} \\
= & \frac{e^{-x}}{x}\left\{1+\frac{\left(\frac{1}{2} x\right)^{2}}{1 ! \rho}+\frac{\left(\frac{1}{2} x\right)^{4}}{2 ! \rho(\rho+1)}+\frac{\left(\frac{1}{2} x\right)^{6}}{3 ! \rho(\rho+1)(\rho+2)}+\cdots\right\} .
\end{aligned}
$$

Hence, the above expression is obviously monotonically decreasing in $\rho$ for a fixed real $x>0$ as each term after the first is a decreasing function of $\rho$.

The rest easily follows Lemma $2 . i i$.

Remark 3 Fix the values of $1 / 2<\rho_{1}<\rho_{2}<+\infty$. Then a combination of formulas (46)-(47) yields that

$$
\int_{0}^{\infty}\left(\tau_{\rho_{1}}(x)-\tau_{\rho_{2}}(x)\right) d x<\infty
$$

Hence, in the case where the r.v. $\mathbf{Y}_{i}$ which emerges in Lemma 2 coincides with the generalized $\rho$-order Feller-Spitzer r.v. of the second type $\mathcal{Y}_{\rho_{i}}($ for $i=1,2)$, the non-negative 
infinitely divisible r.v. $\mathcal{K}\left(=\mathcal{K}_{\rho_{1}, \rho_{2}}\right)$ which is present on the right-hand side of representation (50) is in fact compound Poisson.

For a representative special case for which $\rho_{1}=1$ and $\rho_{2}=2$, the corresponding compound Poisson r.v. $\mathcal{K}\left(=\mathcal{K}_{1,2}\right)$ is identified in Remark 5 of "A case study: properties of the r.v. $\mathcal{Y}_{2}$ " section.

Proposition 5 For an arbitrary fixed $\rho>1 / 2$, the following Poincaré expansion holds as $y \rightarrow+\infty$ for the integral

$$
\int_{y}^{\infty} e^{-x} \frac{I_{\rho-1}(x)}{x^{\rho}} d x \sim \frac{y^{-(\rho-1 / 2)}}{\sqrt{2 \pi}} \sum_{k=0}^{\infty} \frac{(-1)^{k} \Gamma\left(\rho+k-\frac{1}{2}\right)}{\left(\rho+k-\frac{1}{2}\right) \Gamma\left(\rho-k-\frac{1}{2}\right) k !}(2 y)^{-k} .
$$

Proof of Proposition 5 is given in (Paris and Vinogradov (2020b), Lemma 2).

The following assertion generalizes formulas (34)-(35).

Proposition 6 (i) The Lévy measure $v_{\rho}^{(2)}(\{\cdot\})$ on $\mathbf{R}_{+}^{1}$ of positive infinitely divisible r.v. $\mathcal{Y}_{\rho}$ is such that for $y \in \mathbf{R}_{+}^{1}$,

$$
\begin{gathered}
v_{\rho}^{(2)}(\{(y,+\infty)\})=\int_{y}^{\infty} \tau_{\rho}(x) d x=2^{\rho-1} \cdot \Gamma(\rho) \cdot \int_{y}^{\infty} \frac{e^{-x} I_{\rho-1}(x)}{x^{\rho}} d x \\
=-\gamma+y \cdot{ }_{3} F_{3}\left(\begin{array}{c}
1,1, \rho+\frac{1}{2} \\
2,2,2 \rho
\end{array}-2 y\right)-\log (2 y)+\psi(2 \rho-1)-\psi\left(\rho-\frac{1}{2}\right) .
\end{gathered}
$$

(ii) For an arbitrary fixed $\rho>1 / 2$ and as $y \rightarrow+\infty$,

$$
\mathbf{P}\left\{\mathcal{Y}_{\rho}>y\right\} \sim v_{\rho}^{(2)}(\{(y,+\infty)\}) \sim \frac{\Gamma(\rho) \cdot 2^{\rho-1}}{\sqrt{2 \pi}(\rho-1 / 2)} \cdot y^{-(\rho-1 / 2)}
$$

Proof of Proposition 6. (i) The validity of (54) can be established by the use of Mathematica. The analytic proof is given in (Paris and Vinogradov (2020b), Lemma 1).

(ii) Observe that the leading term of the asymptotic series which emerges on the righthand side of (53) is as follows:

$$
\int_{y}^{\infty} e^{-x} \frac{I_{\rho-1}(x)}{x^{\rho}} d x \sim \frac{y^{-\rho+1 / 2}}{\sqrt{2 \pi}(\rho-1 / 2)} \text { as } y \rightarrow+\infty .
$$

Next, combine formulas (3), (46), (56), the leftmost equation in (54), and the well-known fact that the power asymptotics of the function $\tau_{\rho}(x)$ at positive infinity implies the corresponding power asymptotics of its integrated tail. This yields the validity of the rightmost asymptotics in expression (55). The rest follows from an application of (Embrechts et al. (1979), Theorem 1).

The following assertion is of particular value.

Theorem 4 For each $\rho>1 / 2$ and real $\lambda \geq 0$, the negative $\Phi_{\rho}(\lambda)$ of the log-Laplace transform of r.v. $\mathcal{Y}_{\rho}$

$$
\Phi_{\rho}(\lambda)=2^{\rho-1} \cdot \Gamma(\rho) \cdot \int_{0}^{\infty}\left(1-e^{-\lambda x}\right) \cdot \frac{e^{-x} I_{\rho-1}(x)}{x^{\rho}} \cdot d x
$$

admits the following representation in terms of the digamma function $\psi$ and the generalized hypergeometric function ${ }_{3} F_{2}$, which are defined by formulas (105) and (103), respectively: 


$$
\begin{aligned}
& \Phi_{\rho}(\lambda)=\int_{1 /(1+\lambda)}^{1}{ }_{2} F_{1}\left(\frac{1}{2}, 1 ; \rho ; x^{2}\right) \cdot \frac{d x}{x}=\log (1+\lambda) \\
& +\frac{1}{2}\left\{\psi(\rho)-\psi\left(\rho-\frac{1}{2}\right)\right\}-\frac{1}{4 \rho(1+\lambda)^{2}} \cdot{ }_{3} F_{2}\left(\begin{array}{c}
1,1, \frac{3}{2} \\
2,1+\rho
\end{array} \frac{1}{(1+\lambda)^{2}}\right) .
\end{aligned}
$$

Proof of Theorem 4. First, fix $\rho>3 / 2$. For $\lambda \in \mathbf{R}_{+}^{1}$, we follow an argument similar to that used in (Feller (1966a), Section XIII.7) differentiating the integral which emerges on the right-hand side of (57) with respect to $\lambda$. This produces the following identity:

$$
\frac{d}{d \lambda} \int_{0}^{\infty}\left(1-e^{-\lambda x}\right) \cdot \frac{e^{-x} I_{\rho-1}(x)}{x^{\rho}} \cdot d x \equiv \int_{0}^{\infty} e^{-\lambda x} \cdot \frac{e^{-x} I_{\rho-1}(x)}{x^{\rho-1}} \cdot d x .
$$

(For now, the case of $\rho \in(1 / 2,3 / 2]$ has to be excluded, since for such values of $\rho$ the integral which emerges on the right-hand side of (59) diverges).

A subsequent combination of (59) with (1), (17) and (4) stipulates that for each real $\rho>3 / 2$ and $\lambda \geq 0$,

$$
\begin{aligned}
& \Phi_{\rho}(\lambda)=\frac{2(\rho-1)}{2 \rho-3} \cdot \int_{0}^{\lambda} \mathcal{L}_{\mathcal{X}_{\rho-1}}(u) d u \\
& =\int_{0}^{\lambda} \frac{1}{u+1} \cdot{ }_{2} F_{1}\left(\frac{1}{2}, 1 ; \rho ; \frac{1}{(u+1)^{2}}\right) d u:=\mathcal{I} .
\end{aligned}
$$

Next, for a given $\rho>3 / 2$ we will evaluate $\mathcal{I}$ by making the change of variables $x=$ $1 /(u+1)$ to conclude that

$$
\begin{aligned}
& \mathcal{I}=\log (1+\lambda)+\frac{1}{4 \rho} \sum_{k \geq 0} \frac{\left(\frac{3}{2}\right)_{k}(1)_{k}}{(1+\rho)_{k}(2)_{k}}\left(1-\frac{1}{(1+\lambda)^{2 k+2}}\right)=\log (1+\lambda) \\
& +\frac{1}{4 \rho}{ }_{3} F_{2}\left(\begin{array}{l}
1,1,3 / 2 \\
2,1+\rho
\end{array} ; 1\right)-\frac{(1+\lambda)^{-2}}{4 \rho}{ }_{3} F_{2}\left(\begin{array}{l}
1,1,3 / 2 \\
2,1+\rho
\end{array} ; \frac{1}{(1+\lambda)^{2}}\right) .
\end{aligned}
$$

In addition, it is obtained by the use of Mathematica that for $\rho>1 / 2$,

$$
{ }_{3} F_{2}\left(\begin{array}{l}
1,1,3 / 2 \\
2,1+\rho
\end{array} ;\right)=2 \rho \cdot\left\{\psi(\rho)-\psi\left(\rho-\frac{1}{2}\right)\right\} .
$$

In order to establish the validity of (58) for $\rho>3 / 2$, it remains to combine (60)-(62).

Now, although representation (58) has been established for $\rho>3 / 2$ only, the integral (57), which defines the function $\Phi_{\rho}(\lambda)$, converges for $\rho>1 / 2$, and the expression which emerges on the right-hand side of (58) is analytic for $\rho>1 / 2$ when $\lambda \geq 0$. Hence, by analytic continuation, the result (58) holds for all $\rho>1 / 2$.

The following example is in a similar spirit to that of Example 1.

Example 2 For $\rho=1$, a comparison of formula (3) of Definition 2 with representations (6)-(8) of Definition 3 stipulates that in this case,

$$
\Phi_{1}(\lambda) \equiv-\log \mathcal{L}_{\mathcal{Y}_{1}}(\lambda)=\log \left(\lambda+1+\sqrt{\lambda^{2}+2 \lambda}\right)
$$

Hence, the distributions of r.v's $\mathcal{Y}_{1}$ and the canonical Feller-Spitzer r.v. $\mathcal{U}_{1}$ coincide, i.e.,

$$
\mathcal{Y}_{1} \stackrel{\mathrm{d}}{=} \mathcal{U}_{1}\left(\stackrel{\mathrm{d}}{=} \mathcal{X}_{1}\right)
$$


Next, note that the digamma function $\psi$ is defined by formula (105). It is well known that $\psi(1)=-\gamma$ and $\psi(1 / 2)=-\gamma-2 \cdot \log 2$. A combination of these two representations with (63) and (Prudnikov et al. (1990), Section 7.4.2, entry 365) to evaluate the ${ }_{3} F_{2}$ function

$$
{ }_{3} F_{2}\left(\begin{array}{c}
1,1,3 / 2 \\
2,2
\end{array} ; x\right)=-\frac{4}{x} \cdot \log \left(\frac{1+\sqrt{1-x}}{2}\right)
$$

stipulates that

$$
\begin{aligned}
& \Phi_{1}(\lambda) \equiv \log (1+\lambda)+\frac{1}{2} \cdot(\psi(1)-\psi(1 / 2)) \\
& -\frac{1}{4(1+\lambda)^{2}} \cdot{ }_{3} F_{2}\left(\begin{array}{c}
1,1, \frac{3}{2} \\
2,2
\end{array} \frac{1}{(1+\lambda)^{2}}\right) .
\end{aligned}
$$

Evidently, (66) coincides with (58) in the case where $\rho=1$.

It is interesting that although the middle expression which emerges in formula (60) is not defined for $\rho \in(1 / 2,3 / 2]$, but in the case where $\rho=1$ the equality of the left-and right-hand sides on that formula can be derived analytically. In this case,

$$
\mathcal{I}=\int_{1 /(1+\lambda)}^{1}\left(1-x^{2}\right)^{-1 / 2} \frac{d x}{x}=\log \left(\lambda+1+\sqrt{\lambda^{2}+2 \lambda}\right)
$$

(compare to the right-hand side of (63)).

A detailed consideration of the case where $\rho=2$ is presented separately in "A case study: properties of the r.v. $\mathcal{Y}_{2}$ " section. In particular, see Theorem 7 and Remark 6 therein which demonstrate how complex the p.d.f's of the r.v's $\mathcal{Y}_{\rho}$ can be for $\rho \neq 1$.

The following result is of a particular value providing an illustration of the usefulness of numerous relatively recent advances in the theory of infinitely divisible distributions, most of which were summarized in (Steutel and van Harn (2004), Chapter V). To some extent, it can be regarded as a counterpart of Theorem 2 of "Properties of the class $\mathcal{X}_{\rho}$ " section.

Theorem 5 Fix an arbitrary $\rho>1 / 2$. Then

(i) The non-negative r.v. $\mathcal{Y}_{\rho}$ is self-decomposable and absolutely continuous on $\mathbf{R}_{+}^{1}$.

(ii) The limit from the right at the origin for the p.d.f. $p_{\rho}(x)$ of the r.v. $\mathcal{Y}_{\rho}$ is as follows:

$$
p_{\rho}(0+)=\lim _{x \downarrow 0} p_{\rho}(x)=\exp \left\{-\frac{1}{2} \cdot(\psi(\rho)-\psi(\rho-1 / 2))\right\}<+\infty .
$$

(iii) The p.d.f. $p_{\rho}(x)$ decreases monotonically on $(0,+\infty)$ from $p_{\rho}(0+)$ to 0 and satisfies the following integral equation for $x \in \mathbf{R}_{+}^{1}$ :

$$
x \cdot p_{\rho}(x)=2^{\rho-1} \cdot \Gamma(\rho) \cdot \int_{0}^{x} p_{\rho}(x-u) \cdot \frac{e^{-u} \cdot I_{\rho-1}(u)}{u^{\rho-1}} \cdot d u .
$$

(iv) The p.d.f. $p_{\rho}(x)$ has a continuous derivative $p_{\rho}^{\prime}(x)$ on $\mathbf{R}_{+}^{1}$ for which the following functional equation holds:

$$
x \cdot p_{\rho}^{\prime}(x)=2^{\rho-1} \cdot \Gamma(\rho) \cdot \int_{0}^{x} p_{\rho}(x-u) \cdot e^{-u} \cdot \frac{I_{\rho}(u)-I_{\rho-1}(u)}{u^{\rho-1}} \cdot d u .
$$

(v) The limits from the right of the p.d.f. $p_{\rho}(x)$ and its first derivative at the origin are only different by the sign such that

$$
p_{\rho}^{\prime}(0+)=-p_{\rho}(0+)=-\exp \left\{-\frac{1}{2} \cdot(\psi(\rho)-\psi(\rho-1 / 2))\right\} .
$$


(vi) The p.d.f. $p_{\rho}(x)$ exhibits the following power decay as $x \rightarrow+\infty$ :

$$
p_{\rho}(x) \sim \frac{2^{\rho-3 / 2}}{\sqrt{\pi}} \cdot \frac{\Gamma(\rho)}{x^{\rho+1 / 2}} .
$$

\section{Proof of Theorem 5.}

(i) First, by analogy to (Steutel and van Harn (2004), Section V.2), we introduce the canonical density

$$
\mathbf{k}_{\rho}(x):=x \cdot \tau_{\rho}(x), \text { where } x \in \mathbf{R}_{+}^{1} .
$$

Note in passing that in the case where $\rho>3 / 2$,

$$
\mathbf{k}_{\rho}(x) \equiv \frac{2(\rho-1)}{2 \rho-3} \cdot f_{\rho-1}(x),
$$

where $x \in \mathbf{R}_{+}^{1}$, and the p.d.f. $f_{\rho-1}(x)$ can be easily obtained from (1).

The self-decomposability of the r.v. $\mathcal{Y}_{\rho}$ then follows by combining the inequality (110) of "Appendix" section which yields that $\forall \rho>1 / 2$, the canonical density $\mathbf{k}_{\rho}(x)$ is a decreasing function in $\mathbf{R}_{+}^{1}$, with (Steutel and van Harn (2004), Theorem V.2.11). Also, compare (73) with Theorem 2.iii.

Subsequently, a combination of the just established self-decomposability of the class $\mathcal{Y}_{\rho}$, property (47), and (Steutel and van Harn (2004), Theorem V.2.16 and formula (2.28)) implies that $\forall \rho>1 / 2$, the r.v. $\mathcal{Y}_{\rho}$ is absolutely continuous whose p.d.f., which is denoted by $p_{\rho}(x)$, is continuous on $\mathbf{R}_{+}^{1}$.

(ii) For each $\rho>1 / 2$ and $x \in \mathbf{R}_{+}^{1}$, the p.d.f. $p_{\rho}(x)$ is continuous at $x$ and can be evaluated as follows:

$$
\begin{aligned}
p_{\rho}(x)= & \frac{1}{2 \pi} \cdot \int_{-\infty}^{\infty} e^{-i x t} \cdot \exp \left\{-\Phi_{\rho}(-i t)\right\} d t \\
& :=\frac{1}{2 \pi} \cdot \lim _{T \rightarrow+\infty} \int_{-T}^{T} e^{-i x t} \cdot \exp \left\{-\Phi_{\rho}(-i t)\right\} d t .
\end{aligned}
$$

Hereinafter, the factor $\exp \left\{-\Phi_{\rho}(-i t)\right\}=\mathbf{E} \exp \left\{i t \mathcal{Y}_{\rho}\right\}$ is ch.f. of the r.v. $\mathcal{Y}_{\rho}$.

The validity of (74) can be derived with some effort from (Apostol (1957), pp. 498499, Theorem 15.34). Note that since the ch.f. $\exp \left\{-\Phi_{\rho}(-i t)\right\} \notin \mathbf{L}^{1}\left(\mathbf{R}^{1}\right)$, the standard inversion formula of probability theory is not applicable to this class of the discontinuous p.d.f.'s.

Specifically, $p_{\rho}(\cdot)$ is discontinuous at the origin $\forall \rho>1 / 2$. On the other hand, the finiteness of $p_{\rho}(x)$ at zero (as defined by (74)) easily follows from a combination of formula (47) with (Steutel and van Harn (2004), formula (V.2.28)). In addition, a combination of the discontinuous version of the inversion formula with the fact that $p_{\rho}(0-)=0$ (since for each real $x<0$ the p.d.f. $\left.p_{\rho}(x) \equiv 0\right)$ stipulates that

$$
p_{\rho}(0+)=2 \cdot \frac{p_{\rho}(0+)+p_{\rho}(0-)}{2}=2 \cdot \frac{1}{2 \pi} \cdot \int_{-\infty}^{\infty} \exp \left\{-\Phi_{\rho}(-i t)\right\} d t
$$

(compare to (Apostol (1957), p. 499, formulas (79)-(80)) or (Olver et al. (2010), formula (1.14.3))). At the same time, (Steutel and van Harn (2004), Proposition A.3.4) implies that

$$
p_{\rho}(0+):=\lim _{\lambda \rightarrow+\infty}\left(\lambda \cdot \exp \left\{-\Phi_{\rho}(\lambda)\right\}\right) .
$$

The assertion of part (ii) now follows, since in view of (62), the limit that emerges in (76) is finite coinciding with the one that appears in (67). 
(iii) In view of (Steutel and van Harn (2004), Theorem V.2.17), the monotonic decay of $p_{\rho}(x)$ follows from that of the canonical density $\mathbf{k}_{\rho}(x)$ combined with the fact that formula (47) yields that for $\rho>1 / 2$,

$$
\mathbf{k}_{\rho}(0+)=1 \text {. }
$$

The validity of the integral Eq. (68) is then obtained by combining representations (3) and (72) with (Steutel and van Harn (2004), Theorem V.2.16).

(iv) The existence and continuity of $p_{\rho}^{\prime}(x)$ follows from (Steutel and van Harn (2004), p. 237, proof of Theorem V.2.17). Next, observe that for $\rho>1 / 2$, a combination of formula (112) with (Paris and Vinogradov (2020b), the unnumbered formula just below (4.6)) with $v=\rho-1>-1 / 2$ yields that

$$
\begin{aligned}
\frac{d}{d u} \mathbf{k}_{\rho}(u) & =2^{\rho-1} \Gamma(\rho) \cdot \frac{d}{d u} \frac{e^{-u} \cdot I_{\rho-1}(u)}{u^{\rho-1}} \\
& =2^{\rho-1} \Gamma(\rho) \cdot e^{-u} \cdot \frac{I_{\rho}(u)-I_{\rho-1}(u)}{u^{\rho-1}}, \text { where } u \in \mathbf{R}_{+}^{1}
\end{aligned}
$$

The validity of (69) is then obtained by combining formulas (77)-(78) with (Steutel and van Harn (2004), formula (V.2.24)).

(v) It suffices to combine (68)-(69) with (Olver et al. (2010), formula (10.30.1)) and (67) to conclude that for $x \in \mathbf{R}_{+}^{1}$,

$$
\begin{aligned}
p_{\rho}(x) & +p_{\rho}^{\prime}(x)=\frac{2^{\rho-1} \cdot \Gamma(\rho)}{x} \cdot \int_{0}^{x} p_{\rho}(x-u) \cdot \frac{e^{-u} \cdot I_{\rho}(u)}{u^{\rho-1}} \cdot d u \\
& \sim \frac{p_{\rho}(0+)}{4 \rho} \cdot x \rightarrow 0 \text { as } x \downarrow 0 .
\end{aligned}
$$

(vi) The monotonic decay of the p.d.f. $p_{\rho}(x)$ combined with the power asymptotics of the upper tail $\mathbf{P}\left\{\mathcal{Y}_{\rho}>y\right\}$ of the c.d.f. of the r.v. $\mathcal{Y}_{\rho}$ given by (55) implies the corresponding power decay of the p.d.f. per se, as follows from a version of the monotone density theorem presented in (Mikosch (1999), p. 10, Theorem 1.2.9).

Recall that Theorem 1 implies existence of the weak limit for the stochastically decreasing family $\mathcal{X}_{\rho}$, which was identified in Proposition 3 . Similar, the above Theorem 3 implies with some effort existence of the weak limit for the stochastically decreasing family $\mathcal{Y}_{\rho}$ as $\rho \rightarrow+\infty$, which should be a continuous distribution on $\mathbf{R}_{+}^{1}$. In addition, the following assertion, which is a counterpart of Proposition 3, stipulates that for large values of $\rho$, the r.v. $\mathcal{Y}_{\rho}$ can be approximated by the mean 1 exponential r.v. $\mathcal{E}$.

Proposition 7 Suppose that $\rho \rightarrow+\infty$. Then

$$
\mathcal{Y}_{\rho} \stackrel{\mathrm{d}}{\rightarrow} \mathcal{E}
$$

Proof of Proposition 7. We fix $\lambda \in[0,+\infty)$ and combine representations (58)-(60) with (Olver et al. (2010), formula (15.12.2)) to ascertain that as $\rho \rightarrow+\infty$,

$$
\begin{aligned}
\Phi_{\rho}(\lambda)= & \int_{1 /(1+\lambda)}^{1}{ }_{2} F_{1}\left(\frac{1}{2}, 1 ; \rho ; x^{2}\right) \frac{d x}{x}=\int_{1 /(1+\lambda)}^{1}\left(1+\frac{x^{2}}{2 \rho}+\mathcal{O}\left(1 / \rho^{2}\right)\right) \frac{d x}{x} \\
& =\log (1+\lambda)+\mathcal{O}(1 / \rho)
\end{aligned}
$$

The rest coincides with the end of the proof of Proposition 3.i. 
Let us introduce the following quantities:

$$
D_{j}:=\left.(-1)^{j-1} \frac{d^{j}}{d \lambda^{j}} \Phi_{\rho}(\lambda)\right|_{\lambda=0+}, \quad \text { where } 1 \leq j \leq 4 .
$$

The following assertion provides the counterparts of the closed-form representations (40)-(43) (which pertain to the numerical characteristics of r.v. $\mathcal{X}_{\rho}$ ).

Proposition 8 (i) Given $\rho>3 / 2$,

$$
\mu_{\rho}^{(2)}:=\mathbf{E} \mathcal{Y}_{\rho}=2(\rho-1) /(2 \rho-3)
$$

decreases from $+\infty$ to 1 as $\rho$ increases from $3 / 2$ to $+\infty$.

(ii) The second, third and fourth central moments of r.v. $\mathcal{Y}_{\rho}$ admit the following closed-form representations.

For $\rho>5 / 2$, the variance

$$
\left(\sigma_{\rho}^{(2)}\right)^{2}:=\operatorname{Var} \mathcal{Y}_{\rho}=2(\rho-1) /(2 \rho-5)
$$

decreases from $+\infty$ to 1 as $\rho$ increases from $5 / 2$ to $+\infty$.

$$
\begin{aligned}
& \text { Given } \rho>7 / 2 \text {, } \\
& \mu_{3}\left(\mathcal{Y}_{\rho}\right):=\mathbf{E}\left(\left(\mathcal{Y}_{\rho}-\mu_{\rho}^{(2)}\right)^{3}\right)=\frac{8(\rho-1)(\rho-2)}{(2 \rho-5)(2 \rho-7)} .
\end{aligned}
$$

Given $\rho>9 / 2$,

$$
\mu_{4}\left(\mathcal{Y}_{\rho}\right):=\mathbf{E}\left(\left(\mathcal{Y}_{\rho}-\mu_{\rho}^{(2)}\right)^{4}\right)=\frac{24(\rho-1)(\rho-2)}{(2 \rho-7)(2 \rho-9)} .
$$

\section{Proof of Proposition 8.}

(i) With $\Phi_{\rho}(\lambda)$ satisfying the integral representation given in (58) and for $\lambda \in \mathbf{R}_{+}^{1}$, we have

$$
\begin{aligned}
& \frac{d}{d \lambda} \Phi_{\rho}(\lambda)=\frac{d}{d \lambda} \int_{1 /(1+\lambda)}^{1}{ }_{2} F_{1}\left(\frac{1}{2}, 1 ; \rho ; 1 / x^{2}\right) \frac{d x}{x} \\
= & \frac{1}{1+\lambda}{ }_{2} F_{1}\left(\frac{1}{2}, 1 ; \rho ; 1 /(1+\lambda)^{2}\right) .
\end{aligned}
$$

Taking the limit of (83) as $\lambda \downarrow 0$ and combining it with the Gauss summation theorem (see, for example, (Abramowitz and Stegun (1965), formula (15.1.20)) yields that for $\rho>3 / 2$, the first derivative $\Phi_{\rho}^{\prime}(0+)=2(\rho-1) /(2 \rho-3)$.

(ii) Repeated differentiation of (83) using (Abramowitz and Stegun (1965), formula (15.2.1)) produces the three results stated.

An application of the representations of Proposition 8 with the help of Mathematica ascertains that the skewness $\Gamma_{1}^{(2)}(\rho)$ and the excess kurtosis $\Gamma_{2}^{(2)}(\rho)$ of the r.v. $\mathcal{Y}_{\rho}$ are as follows. For $\rho>7 / 2$, the skewness

$$
\Gamma_{1}^{(2)}(\rho)=\frac{D_{3}}{D_{2}^{3 / 2}}=\frac{4(\rho-2)}{2 \rho-7}\left(\frac{2 \rho-5}{2(\rho-1)}\right)^{1 / 2}
$$

decreases from $+\infty$ to 2 as $\rho$ increases from $7 / 2$ to $+\infty$.

In addition, for $\rho>9 / 2$, the excess kurtosis

$$
\Gamma_{2}^{(2)}(\rho)=\frac{D_{4}}{D_{2}^{2}}=\frac{6(\rho-2)}{\rho-1} \frac{(2 \rho-5)^{2}}{(2 \rho-7)(2 \rho-9)}
$$

decreases from $+\infty$ to 6 as $\rho$ increases from $9 / 2$ to $+\infty$. 
Remark 4 (i) Similar to the large- $\rho$ behavior of the numerical characteristics of the class $\left\{\mathcal{X}_{\rho}\right\}$ pointed out in Remark 2 of "Properties of the class $\mathcal{X}_{\rho}$ " section, representations (81), (82), (84) and (85) stipulate that as $\rho \rightarrow+\infty$, the mean, variance, skewness and excess kurtosis of the respective members of the class $\left\{\mathcal{Y}_{\rho}\right\}$ converge to $1,1,2$ and 6 , respectively, which are the values of the corresponding numerical characteristics of the mean 1 exponential r.v. $\mathcal{E}$. Evidently, this observation is consistent with the weak convergence result of Proposition 7.

(ii) In view of (84), the subclass $\left\{\mathcal{Y}_{\rho}, \rho>7 / 2\right\}$ is comprised of the right-skewed distributions. In turn, (85) stipulates that each member of the subclass $\left\{\mathcal{Y}_{\rho}, \rho>9 / 2\right\}$ is leptokurtic.

\section{A case study: properties of the r.v. $\mathcal{Y}_{2}$}

First, Definition 2 yields that the positive infinitely divisible r.v. $\mathcal{Y}_{2}$ is characterized by the following density of its Lévy measure:

$$
\tau_{2}(x):=2 \cdot \frac{e^{-x} I_{1}(x)}{x^{2}}, \text { where } x>0 .
$$

For $\rho=2$, the negative of the log-Laplace transform $\Phi_{2}(\lambda)$ of the r.v. $\mathcal{Y}_{2}$ is simplified as follows (with $w:=1+\lambda$ ):

$$
\begin{gathered}
\Phi_{2}(\lambda)=\log w+\frac{1}{2} \cdot(\psi(2)-\psi(3 / 2))-\frac{1}{8 w^{2}} \\
\times{ }_{3} F_{2}\left(\begin{array}{c}
1,1,3 / 2 \\
2,3
\end{array} ; \frac{1}{w^{2}}\right)=\log (2 w)-\frac{1}{2}-\frac{1}{8 w^{2}} \cdot{ }_{3} F_{2}\left(\begin{array}{c}
1,1,3 / 2 \\
2,3
\end{array} \frac{1}{w^{2}}\right) .
\end{gathered}
$$

It turns out that in the case where $\rho=2$, a simple representation for the generalized hypergeometric function ${ }_{3} F_{2}$ which emerges in (58) holds. Specifically,

$$
{ }_{3} F_{2}\left(\begin{array}{c}
1,1,3 / 2 \\
2,3
\end{array} ; x\right)=-\frac{8}{x}\left(\frac{1-\sqrt{1-x}}{x}-\frac{1}{2}+\log \left(\frac{1+\sqrt{1-x}}{2}\right)\right) .
$$

The validity of (88) easily follows from (Prudnikov et al. (1990), Section 7.4.2, entry 367) or can be derived by using Mathematica.

The following assertion provides a closed-form expression for the Lévy representation of the r.v. $\mathcal{Y}_{2}$ in terms of elementary functions and connects its p.d.f. $p_{2}(x)$ with the members of the class (11).

Proposition 9 Given $\lambda \in[0, \infty)$, the negative of the log-Laplace transform

$$
\begin{aligned}
\Phi_{2}(\lambda) & =\int_{0}^{\infty}\left(1-e^{-\lambda x}\right) \tau_{2}(x) d x=\Phi_{1}(\lambda)+\frac{1}{2} \cdot\left(e^{-2 \Phi_{1}(\lambda)}-1\right) \\
& =\log \left(\lambda+1+\sqrt{\lambda^{2}+2 \lambda}\right)+\lambda^{2}+2 \lambda-(\lambda+1) \cdot \sqrt{\lambda^{2}+2 \lambda} .
\end{aligned}
$$

Proof of Proposition 9. Combine (4), (58) and (88).

In turn, the above representation for $\Phi_{2}(\lambda)$ has a probabilistic interpretation (90) which specifies decomposition (50) in the case where $\mathbf{Y}_{i} \stackrel{\mathrm{d}}{=} \mathcal{Y}_{i}$ (for $i=1,2$ ) and is described in the following remark, which can be viewed as a counterpart to Proposition 2.

Remark 5 The expression $\frac{1}{2} \cdot\left(e^{-2 \Phi_{1}(\lambda)}-1\right)$ which emerges in (89) is recognized as the log-Laplace transform of a particular non-negative compound Poisson r.v. By analogy to representation (50), it is hereinafter denoted by $\mathcal{K}$. Specifically, the r.v. $\mathcal{K}$ admits the following (compound Poisson) representation: 


$$
\mathcal{K} \stackrel{\mathrm{d}}{=} \mathcal{W}_{1}+\ldots+\mathcal{W}_{\mathcal{P} o i s s(1 / 2)}
$$

where positive i.i.d.r.v.' $\left\{\mathcal{W}_{\ell}, \ell \geq 1\right\}$ (which are characterized by formulas (29)-(30) of Proposition 2) are independent of a Poisson counting r.v. Poiss (1/2) with mean $1 / 2$. Hence, the canonical Feller-Spitzer r.v. $\mathcal{U}_{1}$ can be decomposed into the following sum of two nonnegative independent r.v.s:

$$
\mathcal{U}_{1} \stackrel{\mathrm{d}}{=} \mathcal{Y}_{1} \stackrel{\mathrm{d}}{=} \mathcal{Y}_{2}+\mathcal{K} \text {. }
$$

It is relatively easy to find the Lévy representation for the r.v. $\mathcal{K}$. Thus, it follows from a combination of formulas (8), (57) and (89) that for $\lambda \in[0, \infty)$,

$$
\begin{aligned}
& (\lambda+1) \cdot \sqrt{\lambda^{2}+2 \lambda}-\left(\lambda^{2}+2 \lambda\right)=\Phi_{1}(\lambda)-\Phi_{2}(\lambda)=\int_{0}^{\infty}\left(1-e^{-\lambda x}\right) \\
& \times\left(\tau_{1}(x)-\tau_{2}(x)\right) d x=\int_{0}^{\infty}\left(1-e^{-\lambda x}\right) \frac{2 e^{-x}}{x^{2}}\left(\frac{x}{2} \cdot I_{0}(x)-I_{1}(x)\right) d x
\end{aligned}
$$

(compare to (52)). Similar to the proof of Theorem 3, we ascertain that $\forall x \in \mathbf{R}_{+}^{1}$,

$$
\frac{x}{2} \cdot I_{0}(x)-I_{1}(x)>0,
$$

and that

$$
\int_{0}^{\infty}\left(\tau_{1}(x)-\tau_{2}(x)\right) \cdot d x=\int_{0}^{\infty} \frac{2 e^{-x}}{x^{2}} \cdot\left(\frac{x}{2} \cdot I_{0}(x)-I_{1}(x)\right) \cdot d x<\infty
$$

(compare to Remark 3). A subsequent combination of (91)-(93) yields that for $\lambda \in[0, \infty)$, the expression which emerges on the left-hand side of (91) constitutes the negative of the log-Laplace transform of a compound Poisson r.v. with positive summands (i.e., $\mathcal{K}$ ) whose Lévy density equals $\tau_{1}(x)-\tau_{2}(x)$.

Finally, the distribution of the r.v. $\mathcal{K}$ is mixed being comprised of the point mass $1 / \sqrt{e}$ at the origin and the following density component on $\mathbf{R}_{+}^{1}$ :

$$
\frac{e^{-(u+1 / 2)}}{u} \cdot \sum_{k=1}^{\infty} \frac{I_{2 k}(u)}{2^{k-1} \cdot(k-1) !},
$$

which integrates to $1-1 / \sqrt{e}$ over $\mathbf{R}_{+}^{1}$.

Theorem 6 The r.v. $\mathcal{Y}_{2}$ generates the NEF with the domain $\Theta=(-\infty, 0]$ of the canonical parameter $\theta$, which is characterized by the following variance function:

$$
\mathbf{V}_{2}(\mu)=4 \mu^{2} /\left(4-\mu^{2}\right) \text { for } \mu \in(0,2]
$$

in the sense that for $\mu=2, \mathbf{V}_{2}(2)=+\infty$.

Proof of Theorem 6. It follows from (60) that for $\lambda \geq 0$,

$$
\Phi_{2}(\lambda)=2 \int_{0}^{\lambda} \mathcal{L}_{\mathcal{X}_{1}}(u) d u
$$

Since for $\theta \leq 0$, the mean-value mapping equals $\frac{d}{d \theta}\left(-\Phi_{2}(-\theta)\right)$, a combination of (95) with (8) stipulates that it acquires the following form:

$$
2 /\left(1-\theta+\sqrt{\theta^{2}-2 \theta}\right) .
$$

Next, one should use some calculus to establish that the closed-form expression for the inverse of the mean-value mapping (96) is as follows:

$$
1-\mu / 4-1 / \mu \text {, where } \mu \in(0,2] \text {. }
$$


It remains to combine (97) with (Jørgensen (1997), formula (2.18)). (Compare to the derivation of formulas (9)-(10) which pertain to the case of $\rho=1$.)

Theorem 7 The following representation for the p.d.f. $\left\{p_{2}(t), t \in \mathbf{R}_{+}^{1}\right\}$ of the r.v. $\mathcal{Y}_{2}$ in terms of the difference of two convergent series holds:

$$
\begin{aligned}
& p_{2}(t)=\frac{2 t e^{-t}}{\sqrt{\pi}}\left(\sum_{n \geq 0} \frac{(-2)^{n}}{(2 n) !} \sum_{r \geq 0} b_{r}(n)\left(\frac{1}{t} \cdot \frac{d}{d t}\right)^{n}\left[t^{-r-2} I_{2 n+r+1}(t)\right]\right. \\
& \left.-\sum_{n \geq 0} \frac{(-2)^{n}}{(2 n+1) !} \sum_{r \geq 0} b_{r}(n)\left(\frac{1}{t} \cdot \frac{d}{d t}\right)^{n+1}\left[t^{-r-1} I_{2 n+r+2}(t)\right]\right)
\end{aligned}
$$

where $b_{r}(n):=2^{r} \cdot \Gamma\left(n+r+\frac{3}{2}\right) / r$ f for integer $r \geq 0$.

Proof of Theorem 7 is easily obtained by combining Proposition 10 below with (74), (89), and the following representation for the p.d.f. $p_{2}(t)$ as the corresponding inverse Laplace transform:

$$
\begin{aligned}
& p_{2}(t)=\frac{1}{2 \pi} \cdot \int_{-\infty}^{\infty} e^{-i t v} \cdot \exp \left\{-\Phi_{2}(-i v)\right\} d v \\
= & \frac{e^{-t}}{2 \pi i} \int_{c-\infty i}^{c+\infty i} \frac{e^{w t} e^{w \sqrt{w^{2}-1}-w^{2}+1}}{w+\sqrt{w^{2}-1}} \cdot d w .
\end{aligned}
$$

Here, real $t>0$ and $c>1$ are fixed.

Proposition 10 For arbitrary fixed real $t>0$ and $c>1$, the expression which emerges on the right-hand side of (99) does not depend on a particular value of $c>1$ and admits the series representation given on the right-hand side of (98).

Proof of Proposition 10 is given in (Paris and Vinogradov (2020b), Theorem 1).

Next, define

$$
\phi(x):=x \cdot \sqrt{1-x^{2}} \text { with }|x| \leq 1 .
$$

Observe that the trigonometric transformations of the function $\phi(x)$ defined by (100) admit the following representations in terms of Taylor series which are convergent for $|x| \leq 1$ :

$$
\cos \phi(x)=\sum_{n \geq 0} \frac{(-1)^{n}}{(2 n) !} x^{2 n}\left(1-x^{2}\right)^{n}
$$

and

$$
\sin \phi(x)=\sum_{n \geq 0} \frac{(-1)^{n}}{(2 n+1) !} x^{2 n+1}\left(1-x^{2}\right)^{n+1 / 2} .
$$

Remark 6 (i) The closed-form representation (98) for the p.d.f. $p_{2}(t)$ of the r.v. $\mathcal{Y}_{2}$ is quite complicated.

(ii) We were able to verify, both analytically and numerically, that $p_{2}(t)$ does possess the following three properties it has to satisfy:

(ii1) The function $p_{2}(t) \geq 0$ on $\mathbf{R}_{+}^{1}$, since $\sqrt{1-x^{2}} \cos \phi-x \sin \phi \geq 0$ on $x \in[-1,1]$. 
(ii2) The representation for $p_{2}(t)$ which emerges on the right-hand side of formula (98) integrates to 1 over $\mathbf{R}_{+}^{1}$, since

$$
\begin{gathered}
\int_{0}^{\infty} p_{2}(t) \cdot d t=\frac{e}{\pi} \int_{0}^{\infty} \int_{-1}^{1} e^{-(1-x) t} e^{-x^{2}}\left\{\sqrt{1-x^{2}} \cos \phi-x \sin \phi\right\} d x d t \\
=\frac{e}{\pi} \int_{-1}^{1} \frac{e^{-x^{2}}}{1-x}\left\{\sqrt{1-x^{2}} \cos \phi-x \sin \phi\right\} d x=1
\end{gathered}
$$

(ii3) We ascertain the following representation for $p_{2}(t)$ :

$$
\begin{aligned}
& p_{2}(t)=\frac{e}{\pi} \int_{-1}^{1} e^{-(1-x) t} e^{-x^{2}}\left\{\sqrt{1-x^{2}} \cos \phi-x \sin \phi\right\} d x \\
& =\frac{e}{\pi} \cdot \int_{0}^{2} e^{-u t} \mathcal{G}(u) d u \text { with } \mathcal{G}(u)=\frac{4 \sqrt{2}}{3 e} \cdot u^{3 / 2}+\mathcal{O}\left(u^{5 / 2}\right)
\end{aligned}
$$

as $u \downarrow 0$. In turn, a combination of (101) with some algebra implies that

$$
p_{2}(t) \sim \frac{4 \sqrt{2}}{3 \pi} \cdot \int_{0}^{\infty} e^{-u t} u^{3 / 2} d u=\frac{4 \sqrt{2}}{3 \pi} \cdot \Gamma\left(\frac{5}{2}\right) t^{-5 / 2}=\sqrt{\frac{2}{\pi}} t^{-5 / 2} \text { as } t \rightarrow \infty,
$$

which is consistent with representation (71) in the case where $\rho=2$.

\section{Appendix}

In this section, we introduce some relevant special functions as well as review some known and present new results on these functions.

Definition 4 (Gauss hypergeometric function, see, for example, (Olver et al. (2010), formula (15.2.1))). Given arbitrary complex $a$ and $b, c \neq 0,-1,-2, \ldots$, and argument $z \in \mathbb{C}$ with $|z|<1$, the convergent series which emerges on the right-hand side of (102)

$$
{ }_{2} F_{1}(a, b ; c ; z):=\sum_{\ell=0}^{\infty} \frac{(a)_{\ell} \cdot(b)_{\ell}}{(c)_{\ell}} \cdot \frac{z^{\ell}}{\ell !}
$$

generates the so-called Gauss hypergeometric function. Hereinafter, $(a)_{k}=\Gamma(a+k) / \Gamma(a)$ is the Pochhammer symbol.

Definition 5 (Generalized hypergeometric function ${ }_{3} F_{2}$, see (Olver et al. (2010), form. (16.2.1) and case 16.2(iii))). Given arbitrary complex $a, b, c, d \neq 0,-1,-2, \ldots, e \neq$ $0,-1,-2, \ldots$, and argument $z \in \mathbb{C}$ with $|z|<1$, the function

$$
{ }_{3} F_{2}\left(\begin{array}{c}
a, b, c \\
d, e^{\prime}
\end{array} ;\right):=\sum_{\ell=0}^{\infty} \frac{(a)_{\ell} \cdot(b)_{\ell} \cdot(c)_{\ell}}{(d)_{\ell} \cdot(e)_{\ell}} \cdot \frac{z^{\ell}}{\ell !}
$$

is called the generalized hypergeometric function ${ }_{3} F_{2}$.

The infinite series which is present on the right-hand side of (102) can often be simplified. For instance, see (Paris and Vinogradov (2020a), formula (4.1)), and also formulas (65) and (88) for a few relevant special cases of functions ${ }_{2} F_{1}(1 / 2,1 ; c ; z)$ and ${ }_{3} F_{2}\left(\begin{array}{c}a, b, c \\ d, e\end{array} ; z\right)$, respectively. In addition, we refer to (Olver et al. (2010), formula (16.2.1)) and Slater (1960), respectively, for a more general form ${ }_{p} F_{q}$ of a generic member of the class of generalized hypergeometric functions, and for the definition and properties of the confluent hypergeometric function ${ }_{1} F_{1}$, which are also used in this paper. In particular, 
see formula (34) which involves ${ }_{3} F_{3}$, and also formulas (15) and (33) where the function ${ }_{1} F_{1}$ emerges.

The modified Bessel function of the first kind of order $v$ is defined by

$$
I_{v}(t):=\sum_{k=0}^{\infty} \frac{(t / 2)^{2 k+v}}{k ! \cdot \Gamma(k+1+v)} .
$$

In this paper, we also use the digamma function which is denoted by

$$
\psi(z):=\Gamma^{\prime}(z) / \Gamma(z) \text { where } \Re(z)>0
$$

with the Euler-Mascheroni constant

$$
\gamma:=-\psi(1)=-\Gamma^{\prime}(1) .
$$

Lemma 3 Given real $s \in(-1, \rho-1 / 2)$,

$$
\int_{0}^{\infty} x^{s-\rho} \cdot e^{-x} \cdot I_{\rho}(x) d x=\frac{1}{2^{\rho}} \cdot \frac{\Gamma(s+1) \Gamma(\rho-s-1 / 2)}{\Gamma(\rho-s / 2) \Gamma(\rho-s / 2+1 / 2)} .
$$

Proof of Lemma 3. It easily follows from (Abramowitz and Stegun (1965), formula (11.4.13)).

Next, we present the following two previously unknown inequalities involving the modified Bessel function $I_{\nu}(x)$, which seem to be important for the theory of special functions in their own right (see formulas (108)-(109) below). In this paper, they are needed for the derivation of related properties of specific p.d.f.s.

Theorem 8 Fix an arbitrary real $v>-1 / 2$ and assume that the argument $x \in \mathbf{R}_{+}^{1}$. Then the following two inequalities hold:

(i)

$$
\frac{1}{x} I_{\nu}(x) I_{v+1}(x)>I_{v+1}(x)^{2}-I_{v}(x) I_{v+2}(x)
$$

(ii)

$$
I_{v}(x)>\left(1+\frac{2 v+1}{2 x}\right) I_{v+1}(x)
$$

Note that the first inequality (108) stipulates the log-convexity property established in Corollary 1 below, which is needed for the proof of Theorem 2.i. In turn, (109) is employed in the proof of inequality (23) of Theorem 2.ii.

In addition, the second inequality (109) implies that for $x>0$ and $v>-1 / 2$,

$$
I_{v}(x)>I_{v+1}(x),
$$

which was established by Jones (1968). In turn, (110) yields the fulfillment of the representation (22) for $f_{\rho}^{\prime}(x)$, and that all the members of the second class $p_{\rho}(x)$ are self-decomposable.

\section{Proof of Theorem 8.}

(i) From (Olver et al. (2010), formula (10.32.15)), we have the integral representation

$$
I_{\mu}(x) I_{v}(x)=\frac{2}{\pi} \int_{0}^{\pi / 2} I_{\mu+v}(w) \cos (\mu-v) \theta d \theta
$$

for $\mu+v>-1$, where $w:=2 x \cos \theta$. This enables us to express the products of Bessel functions in inequality (108) in terms of a single Bessel function. Then (108) becomes $J>0$, where 


$$
\begin{aligned}
J:=\frac{2}{\pi} \int_{0}^{\pi / 2}\left\{I_{2 v+1}(w) \cos \theta-x I_{2 v+2}(w)(1-\cos 2 \theta)\right\} d \theta \\
=\frac{2}{\pi} \int_{0}^{\pi / 2}\left\{I_{2 v+1}(w) \cos \theta-2 x I_{2 v+2}(w) \sin ^{2} \theta\right\} d \theta .
\end{aligned}
$$

An integration by parts applied to the first integral then yields

$$
J=\frac{2}{\pi} I_{2 v+1}(0)+\frac{4 x}{\pi} \int_{0}^{\pi / 2}\left\{I_{2 v+1}^{\prime}(w)-I_{2 v+2}(w)\right\} \sin ^{2} \theta d \theta .
$$

But

$$
I_{2 v+1}^{\prime}(w)=I_{2 v+2}(w)+\frac{2 v+1}{w} I_{2 v+1}(w)
$$

and so

$$
J=\frac{2}{\pi} I_{2 v+1}(0)+\frac{2(2 v+1)}{\pi} \int_{0}^{\pi / 2} I_{2 v+1}(w) \frac{\sin ^{2} \theta}{\cos \theta} d \theta .
$$

The integral behaves near $\theta=\pi / 2$ like $\cos ^{2 v+1} \theta / \cos \theta$ and so converges provided $v>$ $-1 / 2$ and in this case $I_{2 v+1}(0)=0$. Since the integrand is non-negative in $\theta \in[0, \pi / 2]$ it follows that $J>0$, which concludes the proof of the validity of inequality (108).

(ii) The proof is given in (Paris and Vinogradov (2020b), proof of inequality (4.2) of Theorem 2).

Corollary 1 For an arbitrary fixed real $\rho>1 / 2$, the function $f_{\rho}(x)$ defined by (1) is log-convex on $\mathbf{R}_{+}^{1}$.

Proof of Corollary 1. Denote $f(x):=e^{-x} g(x)$, where

$$
g(x):=x^{-v} I_{v}(x)
$$

Then

$$
f^{\prime}(x)=e^{-x}\left(g^{\prime}(x)-g(x)\right)=e^{-x} x^{-v}\left(I_{v+1}(x)-I_{v}(x)\right)<0
$$

by virtue of (110). Also,

$$
f^{\prime \prime}(x)=e^{-x}\left(g(x)-2 g^{\prime}(x)+g^{\prime \prime}(x)\right),
$$

and so the log-convexity condition $f^{\prime \prime}(x) f(x)>\left(f^{\prime}(x)\right)^{2}$ becomes

$$
\begin{gathered}
e^{-2 x} g(x)\left(g(x)-2 g^{\prime}(x)+g^{\prime \prime}(x)\right)>e^{-2 x}\left(g^{\prime}(x)-g(x)\right)^{2} \\
=e^{-2 x}\left(g(x)^{2}+g^{\prime}(x)^{2}-2 g(x) g^{\prime}(x)\right),
\end{gathered}
$$

to yield

$$
g(x) \cdot g^{\prime \prime}(x)>g^{\prime}(x)^{2} .
$$

Now

$$
g^{\prime}(x)=x^{-v} I_{v+1}(x), \quad \text { and } \quad g^{\prime \prime}(x)=x^{-v-1} I_{v+1}(x)+x^{-v} I_{v+2}(x),
$$

so that the log-convexity condition (113) reduces to the inequality (108).

Ethical approval and consent to participate

Not applicable.

Abbreviations

ch.f.: Characteristic function; i.i.d.r.v.'s: Independent and identically distributed random variables; NEF: Natural exponential family; p.d.f.: Probability density function; p.m.f.: Probability mass function; r.v.: Random variable 


\section{Acknowledgements}

We are thankful to two anonymous referees for a very constructive feedback and numerous suggestions. In particular, we are indebted to referee number one for his/her constructive comments and requests which eventually led to the inclusion of Theorems 1 and 3, Proposition 2, Lemma 2, and Remark 5. WV is grateful to Huaxiong Huang, Konstantin Khanin, Neil Madras and Tom Salisbury for their warm hospitality and thanks the Fields Institute, University of Toronto and York University for continued support.

\section{Authors' contributions}

WV dealt with the overall presentation, proofs of the probability theory results, and drafted the manuscript. RBP dealt with the asymptotics and proofs of the analysis results. Both authors read and approved the final manuscript.

\section{Funding}

WW (the corresponding co-author of the manuscript) was supported by the Ohio University Global Travel Fund.

\section{Availability of data and materials}

Not applicable.

\section{Consent for publication}

Both authors provide consent for publication.

\section{Competing interests}

The authors declare that they have no competing interests.

\section{Author details}

${ }^{1}$ Department of Mathematics, Ohio University, Athens, OH, USA. ${ }^{2}$ Division of Computing and Mathematics, Abertay University, Dundee, UK.

Received: 7 April 2020 Accepted: 12 January 2021

Published online: 04 March 2021

\section{References}

Abramowitz, M., Stegun, I. A.: Handbook of Mathematical Functions with Formulas, Graphs, and Mathematical Tables. Chapman \& Hall, Dover (1965)

Apostol, T. M.: Mathematical Analysis: A Modern Approach to Advanced Calculus. Addison-Wesley, Reading MA (1957) Bertoin, J.: Lévy Processes. Cambridge University Press, Cambridge (1996)

Cohn, H.: On the convergence of stochastically monotone sequences of random variables and some applications. J. Appl. Probab. 18, 592-605 (1981)

Embrechts, P., Goldie, C., Veraverbeke, N.: Subexponentiality and infinite divisibility. Z. Wahrsch. Verw. Gebiete. 49 335-347 (1979)

Feller, W.: An Introduction to Probability Theory and its Applications, vol. II. Wiley, London (1966a)

Feller, W.: Infinitely divisible distributions and Bessel functions associated with random walks. SIAM J. Appl. Math. 14, 864-875 (1966b)

Johnson, N. L., Kotz, S., Balakrishnan, N.: Continuous Univariate Distributions Vol. I. $2^{\text {nd }}$ ed. Wiley, New York (1994)

Jones, A. L.: An extension of an inequality involving modified Bessel functions. J. Math. Phys. 47, 220-221 (1968)

Jørgensen, B.: Exponential dispersion models (with discussion). J. Roy. Stat. Soc. Ser. B. 49, 127-162 (1987)

Jørgensen, B.: The Theory of Dispersion Models. Chapman \& Hall, London (1997)

Kyprianou, A. E.: The Wiener-Hopf factorization. In: Cont, R. (ed.) Encyclopedia of Quantitative Finance, IV Volume Set Vol. 4, pp. 1927-1933. Wiley, Chichester, (2010)

Lee, S., Linton, O., Whang, Y. J.: Testing for stochastic monotonicity. Econometrica. 77, 585-602 (2009)

Letac, G.: Discussion of B. Jørgensen: 'Exponential dispersion models'. J. Roy. Stat. Soc. Ser. B. 49, 154 (1987)

Letac, G.: Lectures on Natural Exponential Families and Their Variance Functions. Monografias de Mathemática, vol. 50. Instituto de Mathemática Pura e Aplicada, Rio de Janeiro (1992)

Letac, G., Mora, M.: Natural real exponential families with cubic variance functions. Ann. Stat. 18, 1-37 (1990)

Mikosch, T.: Regular Variation, Subexponentiality and Their Applications in Probability Theory. EURANDOM Report Vol. 99 Issue 13. Eindhoven University of Technology, Eindhoven (1999)

Olver, F. W. J., Lozier, D. W., Boisvert, R. F., Clark, C. W. (Eds): NIST Handbook of Mathematical Functions. Cambridge University Press, Cambridge (2010)

Paris, R. B., Vinogradov, V.: The evaluation of a weighted sum of Gauss hypergeometric functions and its connection with Galton-Watson processes. Lith. Math. J. 60, 385-395 (2020a)

Paris, R. B., Vinogradov, V.: Some analytical results associated with extensions of the canonical Feller-Spitzer distribution (2020b). arXiv:2008.04797v1 [math.CA]

Prudnikov, A. P., Brychkov, Yu. A, Marichev, O. I.: Integrals and Series: More Special Functions, vol. 3. Gordon \& Breach, New York (1990)

Slater, L. J.: Confluent Hypergeometric Functions. Cambridge University Press, Cambridge (1960)

Spitzer, F.: Principles of Random Walk. D. Van Nostrand Co. Inc., Princeton (1964)

Stewart, W. J.: Probability, Markov Chains, Queues and Simulation: the Mathematical Basis of Performance Modeling. Princeton University Press, Princeton (2009)

Steutel, F. W., van Harn, K.: Infinite Divisibility of Probability Distributions on the Real Line. Dekker, New York (2004)

Watson, G. N.: A Treatise on the Theory of Bessel Functions, $2^{\text {nd }}$ ed. Cambridge University Press, Cambridge (1952)

\section{Publisher's Note}

Springer Nature remains neutral with regard to jurisdictional claims in published maps and institutional affiliations. 